\title{
Gaussian Process Assisted Stochastic Dynamic Analysis with Applications to Near-Periodic Structures
}

\author{
Tanmoy Chatterjee ${ }^{a}$, Danilo Karličića ${ }^{\mathrm{b}, *}$, Sondipon Adhikari $^{\mathrm{a}}$, Michael I. Friswell ${ }^{\mathrm{a}}$ \\ ${ }^{a}$ College of Engineering, Swansea University, Bay Campus, SA1 8EN, United Kingdom \\ ${ }^{b}$ Mathematical Institute of the Serbian Academy of Sciences and Arts, Kneza Mihaila 36, Belgrade, Serbia
}

\begin{abstract}
This paper characterizes the stochastic dynamic response of periodic structures by accounting for manufacturing variabilities. Manufacturing variabilities are simulated through a probabilistic description of the structural material and geometric properties. The underlying uncertainty propagation problem has been efficiently carried out by functional decomposition in the stochastic space with the help of Gaussian Process (GP) meta-modelling. The decomposition is performed by projected the response onto the eigenspace and involves a nominal number of actual physics-based function evaluations (the eigenvalue analysis). This allows the stochastic dynamic response evaluation to be solved with low computational cost. Two numerical examples, namely an analytical model of a damped mechanical chain and a finite-element model of multiple beam-mass systems, are undertaken. Two key findings from the results are that the proposed GP based approximation scheme is capable of (i) capturing the stochastic dynamic response in systems with well-separated modes in the presence of high levels of uncertainties (up to 20\%), and (ii) adequately capturing the stochastic dynamic response in systems with multiple sets of identical modes in the presence of $5-10 \%$ uncertainty. The results are validated by Monte Carlo simulations.
\end{abstract}

Keywords: Mechanical chain, Multiple beam-mass system, Mode degeneration, Eigensolution, Gaussian Process.

\section{Introduction}

There has been considerable attention towards analyzing periodic structures in an attempt to explore and engineer their structural properties which could be tailored for enhanced performance in engineering applications such as vibration attenuation and energy harvesting $[1,2,3]$. However, the need for ideal periodic architectures introduces multiple challenges in the fabrication precision of these periodic structures, which often suffer from anomalies related to the manufacturing process $[4,5]$. Consequently, realizing a series of perfectly identical unit cells become unrealistic in the presence of inevitable manufacturing uncertainties that eventually lead to undesirable performance variations. Also, it has been shown [6] that randomness may be harnessed to enhance the performance of periodic structures.

\footnotetext{
${ }^{*}$ Corresponding author

Email address: danilo.karlicic@swansea.ac.uk (Danilo Karličić )
} 
Driven by the above motivating reasons, the primary goal of this work is to develop a computational framework for the stochastic dynamic analysis of meta-structures to study the effect of uncertainties on the targeted performance. The present study is timely as the underlying assumption in most existing studies is that the dynamic response of the model is obtained for a particular set of physical parameters which are precisely known. This assumption is not necessarily valid, especially for an industrially manufactured product with multiple sources of uncertainties. Thus, for the deterministic model to accurately simulate the system physics, the quantification of the variation in the response is equally important, if not more so [7]. Babaa et al. [8] recently illustrated that perturbations in the input parameters of elastic meta-materials can cause significantly variation in the output response, emphasizing the need for stochastic response analysis.

Uncertainty Quantification (UQ) aims to characterize the relevant uncertainties in physical models from the available measurement data and efficiently propagate these uncertainties for the quantitative validation of the model [9]. UQ has received considerable attention over the last two decades, however, it remains a gruelling challenge to efficiently propagate uncertainties through systems characterized by a large number of uncertain sources where the curse-of-dimensionality is an unresolved problem [10]. Additionally, the development of non-intrusive uncertainty propagation techniques is vital as the analysis of multi-disciplinary systems often requires the use of sophisticated deterministic solvers which cannot be readily modified to incorporate the necessary propagation tools [11].

Monte Carlo Simulation (MCS) has been employed as the primary modus operandi for uncertainty quantification [12]. However, it is well established that MCS leads to computational inefficiency for large-scale systems due to the slow rate of convergence. Several improvements to conventional MCS, such as importance sampling, directional simulation and subset simulation, have been proposed $[13,14]$. There has been increasing recent interest in developing alternative numerical methods that are more efficient than these sampling techniques.

In this context, surrogate-based UQ has gained tremendous popularity due to their computational efficiency compared to sampling-based approaches [15]. Surrogate-based approaches are most suitable when the response function has an implicit form and evaluation is required by numerical approaches such as the finite element (FE) method. Surrogate modelling generates an algebraic approximation to the input-response map of the system. These techniques approximate the underlying computational model in a sample space and thereby reduce the simulation time significantly. For an overview of the various types of surrogates, readers are referred to $[16,17,18,19]$. The Gaussian process (GP) [20,21] is one surrogate modelling technique which has been widely acclaimed for emulating black-box functions successfully in complex computationally intensive tasks such as reliability analysis, optimization under uncertainty, sensitivity analysis, moment estimation and so on. The GP has been implemented in this present work to capture the stochastic dynamic response in a cost-effective manner by replacing high-fidelity physics-based computations. In doing so, the focus has been to accurately capture the input-response behaviour trend with minimum computational effort. Some recent applications of GP in stochastic structural dynamic analysis can be found in $[22,23,24,25,26,27]$. 
One of the earliest notable contributions that analyzed structural models as periodic structures, was by Mead and co-workers [28]. The main emphasis in the early works was wave propagation applied to one-dimensional $[29,30,31]$ and two-dimensional periodic structures [32], consisting of beam and plate models. Moreover, it was shown that in a heterogeneous structured medium, such as beams and plates, dispersion occurs due to the presence of physical boundaries. It was further demonstrated that band-gaps existed as frequency intervals within which waves decay exponentially. In the present work, a model consisting of multiple coupled beam is used, which represents a model of periodically distributed beams connected by an elastic medium as a mechanical chain, in which the free vibration and damped frequency responses are analyzed.

The problem of mechanical chain and multiple connected structural elements have a long history and wide applications in mechanical, civil and aerospace engineering. One of the first problems and application of mechanical chains in mechanical engineering goes back to the 1950s in the papers of Rašković [33, 34]. In the same decade, Dublin and Friedrich [35] investigated the dynamical behaviour of two beams connected by a spring-damper system. Most of their following studies were based on the free and forced vibration of the two coupled beam system with elastic and viscoelastic properties, where the authors analyzed the natural frequencies and amplitude ratios $[36,37,38,39,40]$. In the last decade, the problem of multiple-beam systems connected with elastic layers has received great attention from the research community. Kelly et al. [41, 42] analyzed the free vibration of multiple connected beam systems using analytical and Rayleigh-Ritz approximation methods. Mao [43] proposed the application of the Adomian modified decomposition method to analyze the free vibration of multiple connected beams with elastic boundary conditions. Stojanović and Kozić [44] applied higher-order beam theories, such as Timoshenko and Reddy, to model a multiple connected thick beam system to determine the natural frequencies. In papers of Karličić et al. [45, 3], the influence of nonlocal parameters on the free vibration of multiple connected nanobeam systems was analyzed with the help of analytical and approximate methods. Recently, Paunović et al.[2] analyzed the damped vibration of a multiple fractional viscoelastic beam system by Galerkin approximation. Pavlović et al. [46] studied the stochastic stability of a nonlocal multiple nanobeam system subjected to time-varying axial load.

In the above papers investigating applications of mechanical chain and coupled beam mass systems, the material and geometrical characteristics of the system were assumed to be known (constants) and hence there was no account of any resulting variation in the dynamic response. Therefore, in this paper, we present a rigorous analysis of the response variation in mechanical chain and coupled beam mass systems due to manufacturing anomalies. Hence, the following issues are addressed,

- Methodology: To capture the response fluctuation, a functional decomposition in stochastic space is performed using a GP based meta-modelling technique. The non-intrusive framework results in a substantial reduction in the computational effort by replacing the high-fidelity stochastic analysis. 
- Application: The robustness of the proposed approach is investigated by assessing its ability to capture the variation in modal space. This is illustrated by critical and commonly encountered scenarios, such as high levels of input uncertainty in chain systems having well-separated modes and a moderate level of input uncertainty in coupled beam mass systems having multiple sets of identical modes (featuring mode degeneration).

\section{Stochastic analysis}

\subsection{General problem statement}

Considering a mechanical system whose behaviour can be modelled by a set of governing equations, for instance partial differential equations, and utilizing some suitable solution scheme, the computational model can be represented as

$$
\mathbf{y}=\mathbf{M}(\mathbf{x})
$$

where $\mathbf{x} \in \mathbb{R}^{M}$ is a vector of input parameters of the model. These parameters may be related to the system geometry, material constitutive behaviour or the applied loading conditions. $\mathbf{y} \in \mathbb{R}^{Q}$ is the vector of response quantities which generally consist of the following,

- The displacement response or its associated components,

- The strain and stress component tensor at specified locations,

- The plastic strain and other internal damage indicators,

- Spatial and temporal variations of one or a combination of the above response parameters.

In this work, the focus is a non-intrusive approach for uncertainty quantification, in which the computational model, M, is considered as a black box, i.e. it cannot be modified by the analyst but only run for a set of input parameters. Also, if a set of input parameters is fed into the model, a unique response vector of interest is obtained. Thus, the model $\mathbf{M}$ is purely deterministic, i.e. simulating the model twice utilizing the same input vector will yield the same output results. Moreover, the uncertainties in the model input parameters can be modelled by a random vector $\mathbf{x} \in \mathbb{R}^{M}$ and the associated probability density function $f_{x}(\mathbf{x})$. The classical approach utilizes statistical inference techniques, for example the maximum likelihood principle [21], to fit the best distribution such as Gaussian, lognormal, Gumbel, Beta, etc. Finally, the best distribution is selected by utilizing criteria such as the Akaike or Bayesian information criteria [47]. In cases where little data is available, a prior expert judgement can be combined with measurements through the Bayesian statistics framework [48]. When no data is available, the principle of maximum entropy can be utilized [49]. 


\subsection{Dynamic analysis of finite element models in the frequency domain}

In the stochastic finite element literature, the procedures to obtain the discretized random configuration of the governing partial differential equations are well established. The multi degrees of freedom structural vibration problem can be expressed, using the FE method, as,

$$
\mathbf{M}(\theta) \ddot{\mathbf{u}}(t)+\mathbf{C}(\theta) \dot{\mathbf{u}}(t)+\mathbf{K}(\theta) \mathbf{u}(t)=\mathbf{f}(t)
$$

The system matrices, mass $\mathbf{M}(\theta)$, stiffness $\mathbf{K}(\theta)$ and damping $\mathbf{C}(\theta)$, are random in nature, expressed as a function of the stochastic parameters $\theta$. It is to be noted that uncertainty in the above system matrices is propagated from the random material and geometrical parameters (denoted as $\theta$ ) at the finite element level. $\mathbf{f}$ denotes the applied force which may be deterministic or random and $t$ represents the time. The displacement is represented by $\mathbf{u}(t)$ and the first and second derivatives of the displacement with respect to time are denoted as $\dot{\mathbf{u}}(t)$ and $\ddot{\mathbf{u}}(t)$, respectively. In this paper, the damping is assumed to be proportional, although the proposed approximation scheme is generalized and can be used for non-proportional and other complex damping models.

The Fourier transform of Eq. (2) is used to evaluate the dynamic response in the frequency domain, which yields,

$$
\left[-\omega^{2} \mathbf{M}(\theta)+\mathbf{C}(\theta)+\mathbf{K}(\theta)\right] \tilde{\mathbf{u}}(\omega, \theta)=\tilde{\mathbf{f}}(\omega)
$$

where $\tilde{\mathbf{u}}$ and $\tilde{\mathbf{f}}$ are the dynamic displacement response and forcing in the frequency domain. The coefficient matrix in

Eq. (3), also known as the dynamic stiffness matrix $\mathbf{D}$, is a function of the random parameters $\theta$. $\mathbf{D}$ has to be computed for each forcing frequency and every random realization. This makes the direct mapping of stochastic input parameters to the frequency response directly in the physical space computationally cumbersome.

\section{Proposed projection-based approximation of the dynamic response}

\subsection{Projection methods}

The solution by direct Monte Carlo simulation (dMCS) can be considered as the benchmark to obtain the stochastic dynamic response as,

$$
\tilde{\mathbf{u}}_{d M C S}(\omega, \theta)=\left[-\omega^{2} \mathbf{M}(\theta)+\mathbf{C}(\theta)+\mathbf{K}(\theta)\right]^{-1} \tilde{\mathbf{f}}(\omega)
$$

for each forcing frequency and random realization. The dynamic response can be represented as the projection onto a stochastic basis with stochastic coefficients given by

$$
\tilde{\mathbf{u}}(\omega, \theta)=\sum_{j=1}^{N} \alpha_{j}(\omega, \theta) \mathbf{b}_{j}(\theta)
$$

where $\alpha_{j}(\omega, \theta) \in \mathbb{C}$ represents the random scalars and $\mathbf{b}_{j}(\theta) \in \mathbb{C}^{N}$ denotes the stochastic basis. The random scalars and stochastic basis can be obtained by various numerical techniques [50]. However, the method can be numerically 
unstable due to the non-uniqueness of the solution. Thus, to avoid this instability, the residual can be computed in terms of the $L_{2}$ relative error, and hence the expression for the approximate error is obtained as

$$
\hat{\epsilon}(\omega, \theta)=\tilde{\mathbf{u}}(\omega, \theta)-\tilde{\mathbf{u}}_{d M C S}(\omega, \theta)
$$

Since a closed form expression of the error can be derived in the domain space of $\mathbf{D}(\omega, \theta)$, the residual can be re-written as,

$$
\mathbf{r}(\omega, \theta)=\mathbf{D}(\omega, \theta) \tilde{\mathbf{u}}(\omega, \theta)-\tilde{\mathbf{f}}(\omega)=\mathbf{D}(\omega, \theta)\left[\tilde{\mathbf{u}}(\omega, \theta)-\tilde{\mathbf{u}}^{*}(\omega, \theta)\right]
$$

where $\tilde{\mathbf{u}}^{*}(\omega, \theta)$ represents the true solution which cannot be evaluated exactly. In this case, we can treat the solution $\tilde{\mathbf{u}}_{d M C S}(\omega, \theta)$ obtained by dMCS as the benchmark. By denoting $\mathbf{e}(\omega, \theta)=\tilde{\mathbf{u}}(\omega, \theta)-\tilde{\mathbf{u}}^{*}(\omega, \theta)$ as the true error, this error can be written as

$$
\mathbf{e}(\omega, \theta)=\mathbf{D}^{-1}(\omega, \theta) \mathbf{r}(\omega, \theta)
$$

However, $\mathbf{e}(\omega, \theta)$ cannot be computed exactly therefore an approximate error indicator is required. Thus, a bilinear form is defined as $\overline{\mathbf{D}}(\mathbf{c}, \mathbf{d})=\langle\mathbf{D}(\omega, \theta) \mathbf{c}(\omega, \theta), \mathbf{d}(\omega, \theta)\rangle$, where $\langle\cdot, \cdot\rangle$ represents an inner product in $L_{2} \times \mathbb{R}^{N}$. Then, from Eq. (8),

$$
\overline{\mathbf{D}}(\mathbf{e}, \hat{\epsilon})=R_{\hat{\epsilon}} \quad \text { where } \quad R_{\hat{\epsilon}}=\langle\mathbf{r}(\omega, \theta), \hat{\epsilon}(\omega, \theta)\rangle
$$

Using the Cauchy-Schwarz inequality, one obtains

$$
|\overline{\mathbf{D}}(\mathbf{e}, \hat{\epsilon})|^{2} \leq \overline{\mathbf{D}}(\mathbf{e}, \mathbf{e}) \overline{\mathbf{D}}(\hat{\epsilon}, \hat{\epsilon})=\|\mathbf{e}\|_{E}\|\hat{\epsilon}\|_{E}
$$

where $\|\cdot\|_{E}$ represents the norm consistent with the bilinear form $\overline{\mathbf{D}}(\cdot, \cdot)$ on $L_{2} \times \mathbb{R}^{N}$ (analogous to the elastic potential energy norm for structural dynamic systems). From Eqs. (9) and (10), one can obtain

$$
\left|R_{\hat{\epsilon}}\right|^{2} /\|\hat{\epsilon}\|_{E}<\|\mathbf{e}\|_{E}
$$

Eq. (11) corresponds to the lower bound of the true error $\mathbf{e}(\omega, \theta)$ in terms of the approximate error indicator $\hat{\epsilon}(\omega, \theta)$. The equality holds only under special circumstances detailed in [51]. However, the computational effort for the above approach is significantly higher than that required to obtain the benchmark solution. Therefore, a more computationally efficient strategy is proposed in the following sections.

\subsection{Approximating the stochastic eigensolution}

To implement an efficient approach, the generalized eigensolutions are obtained using a meta-model for the undamped case as follows

$$
\mathbf{K}(\theta) \hat{\boldsymbol{\phi}}_{k}(\theta)=\hat{\lambda}_{k}(\theta) \mathbf{M}(\theta) \hat{\boldsymbol{\phi}}_{k}(\theta) \quad \text { for } \quad k=1,2, \ldots, N
$$


where $\hat{\lambda}_{k}(\theta)$ and $\hat{\phi}_{k}(\theta)$ are the $k^{\text {th }}$ undamped eigenvalue and eigenvector predicted by the meta-model, respectively. For concise representation, they can be defined as,

$$
\begin{array}{r}
\hat{\boldsymbol{\Omega}}^{2}(\theta)=\operatorname{diag}\left[\hat{\lambda}_{1}(\theta), \hat{\lambda}_{2}(\theta), \ldots, \hat{\lambda}_{n}(\theta)\right] \in \mathbb{R}^{N \times N} \\
\hat{\boldsymbol{\Phi}}(\theta)=\left[\hat{\boldsymbol{\phi}}_{1}(\theta), \hat{\boldsymbol{\phi}}_{2}(\theta), \ldots, \hat{\boldsymbol{\phi}}_{n}(\theta)\right] \in \mathbb{R}^{N \times N}
\end{array}
$$

The estimated eigenvalues are arranged in ascending order so that $\hat{\lambda}_{1}(\theta)<\hat{\lambda}_{2}(\theta)<\ldots<\hat{\lambda}_{n}(\theta)$ and the corresponding eigenvectors are mass normalised and arranged in the same order. The following relations can be established

$$
\begin{array}{r}
\hat{\boldsymbol{\Phi}}^{T}(\theta) \mathbf{M}(\theta) \hat{\boldsymbol{\Phi}}(\theta)=\mathbf{I} \\
\hat{\boldsymbol{\Phi}}^{T}(\theta) \mathbf{K}(\theta) \hat{\boldsymbol{\Phi}}(\theta)=\hat{\boldsymbol{\Omega}}^{2}(\theta)
\end{array}
$$

The system response can be obtained by projecting on the undamped eigenvectors as the undamped eigenvectors form a complete basis.

The approximate dynamic response in the frequency domain can be obtained as

$$
\tilde{\mathbf{u}}(\omega, \theta)=\hat{\boldsymbol{\Phi}}(\theta)\left[-\omega^{2} \mathbf{I}(\theta)+2 i \omega \boldsymbol{\xi} \hat{\mathbf{\Omega}}(\theta)+\hat{\mathbf{\Omega}}^{2}(\theta)\right]^{-1} \hat{\boldsymbol{\Phi}}^{T}(\theta) \tilde{\mathbf{f}}(\omega)
$$

where $\boldsymbol{\xi}$ is the diagonal matrix of modal damping ratios for the assumed proportional damping model. Here we assume that these damping ratios are fixed. The above expression can be rewritten as a summation of contributions from each of the estimated $N$ vibration modes of the dynamic system as

$$
\tilde{\mathbf{u}}(\omega, \theta)=\sum_{j=1}^{N} \alpha_{j}(\omega, \theta) \mathbf{b}_{j}(\theta)=\sum_{j=1}^{N}\left(\frac{\hat{\phi}_{j}^{T}(\theta) \tilde{\mathbf{f}}(\omega)}{\hat{\lambda}_{j}(\theta)-\omega^{2}+2 i \sqrt{\hat{\lambda}_{j}(\theta)} \omega \xi_{j}}\right) \hat{\phi}_{j}(\theta)
$$

In Eq. (16), the random scalars $\alpha_{j}(\omega, \theta)=\left(\frac{\hat{\phi}_{j}^{T}(\theta) \tilde{\mathbf{f}}(\omega)}{\hat{\lambda}_{j}(\theta)-\omega^{2}+2 i \sqrt{\hat{\lambda}_{j}(\theta) \omega \xi_{j}}}\right)$ are projected onto the space spanned by $\hat{\boldsymbol{\phi}}_{j}(\theta)$ to obtain the approximate dynamic response.

Figure 1 shows a schematic of the proposed approximation framework, illustrating the input uncertainty propagation and quantification of the dynamic response. The schematic highlights that the need to compute the dynamic response using the high-fidelity FE model for every forcing frequency $\omega$ is eliminated. Instead, the material and geometrical uncertainties at the FE level are mapped onto the undamped eigensolutions using a small number of high-fidelity physics-based computations and then the low-fidelity meta-model based approximation of frequency responses is performed. The Gaussian Process has been used as the meta-modelling technique in this work, which is presented briefly in the next section.

\subsection{Gaussian Process modelling}

The Gaussian Process (GP) is a stochastic process which stipulates probability distributions over functions. Originally the GP was developed as a spatial interpolation technique in the field of geostatistics [52]. GP is also known as Kriging in several disciplines $[53,54,55,56]$. Considering an independent variable $\mathbf{x} \in \mathbb{R}^{d}$ and function 


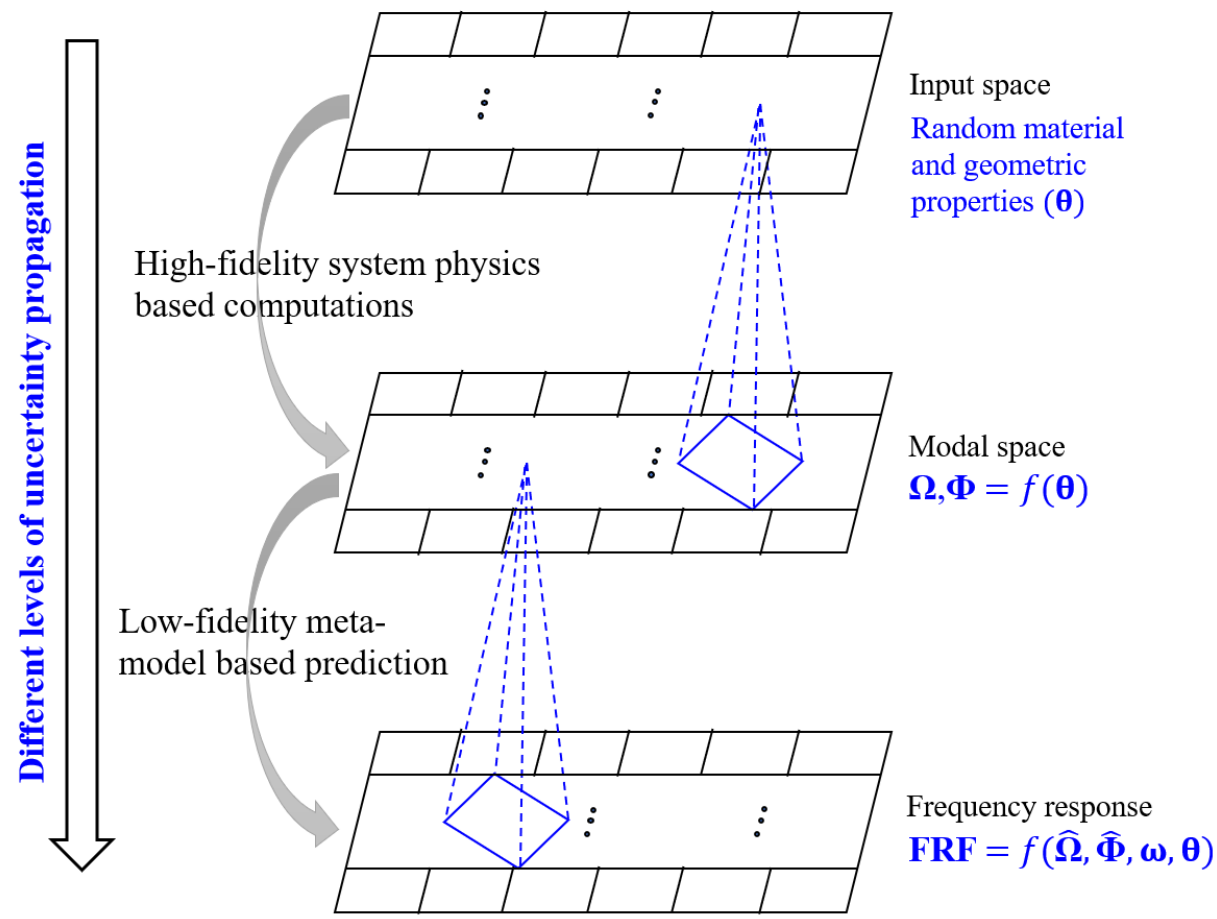

Figure 1: A schematic representation of the computational framework of the approximation strategy to evaluate the stochastic dynamic behaviour.

$g(\mathbf{x})$ such that $g: \mathbb{R}^{d} \rightarrow \mathbb{R}$, a GP over $g(\mathbf{x})$ with mean $\mu(\mathbf{x})$ and covariance function $\kappa\left(\mathbf{x}, \mathbf{x}^{\prime} ; \theta\right)$ can be defined as

$$
\begin{array}{r}
g(\mathbf{x}) \sim G P\left(\mu(\mathbf{x}), \kappa\left(\mathbf{x}, \mathbf{x}^{\prime} ; \Theta\right)\right), \\
\mu(\mathbf{x})=\mathbb{E}[g(\mathbf{x})] \\
\kappa\left(\mathbf{x}, \mathbf{x}^{\prime} ; \Theta\right)=\mathbb{E}\left[(g(\mathbf{x})-\mu(\mathbf{x}))\left(g\left(\mathbf{x}^{\prime}\right)-\mu\left(\mathbf{x}^{\prime}\right)\right)\right]
\end{array}
$$

where $\Theta$ denotes the hyperparameters of the covariance function $\kappa$. The choice of the covariance function $\kappa$ allows the incorporation of any prior knowledge about $g(\mathbf{x})$ (for instance, periodicity, linearity, smoothness) [57]. The following squared exponential (Gaussian) covariance function has been used in this study.

$$
\kappa\left(\mathbf{x}, \mathbf{x}^{\prime}\right)=\sigma_{g}^{2} \exp \left[-\sum_{i=1}^{d} \frac{\left(x(i)-x^{\prime}(i)\right)^{2}}{2 r_{i}^{2}}\right]
$$

where $\left\{\sigma_{g}, r_{1}, \ldots, r_{d}\right\}=\Theta$ are the hyperparameters of the covariance function, and $x(i)$ denotes the $i^{\text {th }}$ element of $\mathbf{x}$.

The most general form of GP, which is called Universal Kriging, has been used in this study [58]. Universal Kriging can be represented by second-order polynomial trend functions and GP as

$$
\mathbf{Y}(\mathbf{x})=\sum_{j=1}^{p} \beta_{j} \mathbf{f}_{j}(\mathbf{x})+\mathbf{Z}(\mathbf{x})
$$

where $\boldsymbol{\beta}=\left\{\beta_{j}, j=1, \ldots, p\right\}$ is the vector of unknown coefficients and $\mathbf{F}=\left\{\mathbf{f}_{j}, j=1, \ldots, p\right\}$ are the polynomial basis functions. $\mathbf{Z}(\mathbf{x})$ is the GP with zero mean and autovariance $\operatorname{cov}\left[\mathbf{Z}(\mathbf{x}), \mathbf{Z}\left(\mathbf{x}^{\prime}\right)\right]=\sigma^{2} \mathbf{R}\left(\mathbf{x}, \mathbf{x}^{\prime}\right)$, where $\sigma^{2}$ is the 
process variance and $\mathbf{R}\left(\mathbf{x}, \mathbf{x}^{\prime}\right)$ is the autocorrelation function.

The parameters $\boldsymbol{\beta}$ and $\sigma^{2}$ can be estimated by the maximum likelihood estimate (MLE) [21]. Now the prediction response for a test point requires three conditions to be satisfied, which are linearity in terms of the observed data, unbiasedness and minimal variance. The prediction mean and variance by GP can be obtained as

$$
\begin{gathered}
\mu_{\hat{Y}}(\mathbf{x})=\boldsymbol{f}(\mathbf{x})^{T} \hat{\boldsymbol{\beta}}+\mathbf{r}^{T} \mathbf{R}^{-1}(\mathbf{y}-\mathbf{F} \hat{\boldsymbol{\beta}}) \\
\sigma_{\hat{Y}}^{2}(\mathbf{x})=\hat{\sigma^{2}}\left[1-\mathbf{r}^{T} \mathbf{R}^{-1} \mathbf{r}+\mathbf{u}^{T}\left(\mathbf{F}^{T} \mathbf{R}^{-1} \mathbf{F}\right)^{-1} \mathbf{u}\right]
\end{gathered}
$$

where $\mathbf{u}=\mathbf{F}^{T} \mathbf{R}^{-1} \mathbf{r}-\mathbf{R}$ and $\mathbf{r}$ is the autocorrelation between the unknown point $\mathbf{x}$ and each point of the observed data set. It is possible to derive confidence bounds on the prediction by GP. The variance information is often used as an error measure of the epistemic uncertainty of the meta-model due to the sparsity of data [59, 60]. This feature has led to the development of adaptive error based sampling schemes for improving the accuracy of the meta-model [61, $62]$.

Figure 2 gives a flow chart of the overall computational framework of the proposed approximation strategy using GP. It is observed that a nominal number of eigenvalue analyses of the actual system have to be performed to train the GP. Once this is done, the modal solutions predicted by GP can be utilized to compute the dynamic response in the frequency domain. Thus, the variation in the frequency response can be quantified using limited computational effort depending upon the level of approximation accuracy desired. The computational cost of the proposed approximation scheme is proportional to $\left(n_{\text {samp }} \times N^{2}\right)$, where $n_{\text {samp }}$ is the number of training points for the GP, the cost of a single eigenvalue analysis performed using the built-in function eig() in MATLAB scales with $N^{2}$ and $N$ represents the number of DOFs. The details of eigenvalue algorithms (generalized Schur decomposition and Cholesky factorization) and the handling of sparse matrices for eigenvalue analysis in MATLAB can be found in [63, 64].

The above computational cost is significantly more efficient than computing the stochastic dynamic response $(N$ $\times N$ matrix) for every forcing frequency and random realization, directly in physical space. Also, compared to dMCS, the proposed approximation methodology utilises $n_{\text {samp }} / n_{M C S}$ computational effort in evaluating the dynamic response, where $n_{M C S}$ denotes the number of dMCS. This computational efficiency may prove to be substantial for large-scale FE models as a single simulation may require significant CPU time and it must be repeated for every stochastic realization to simulate the uncertainties present within the element (local) level. In most structural applications, it is found that the dynamics can be captured by a relatively small number of linear modes. Thus, further computational efficiency can be achieved by model-order reduction of the FE model, however, this is not within the scope of the present work. 


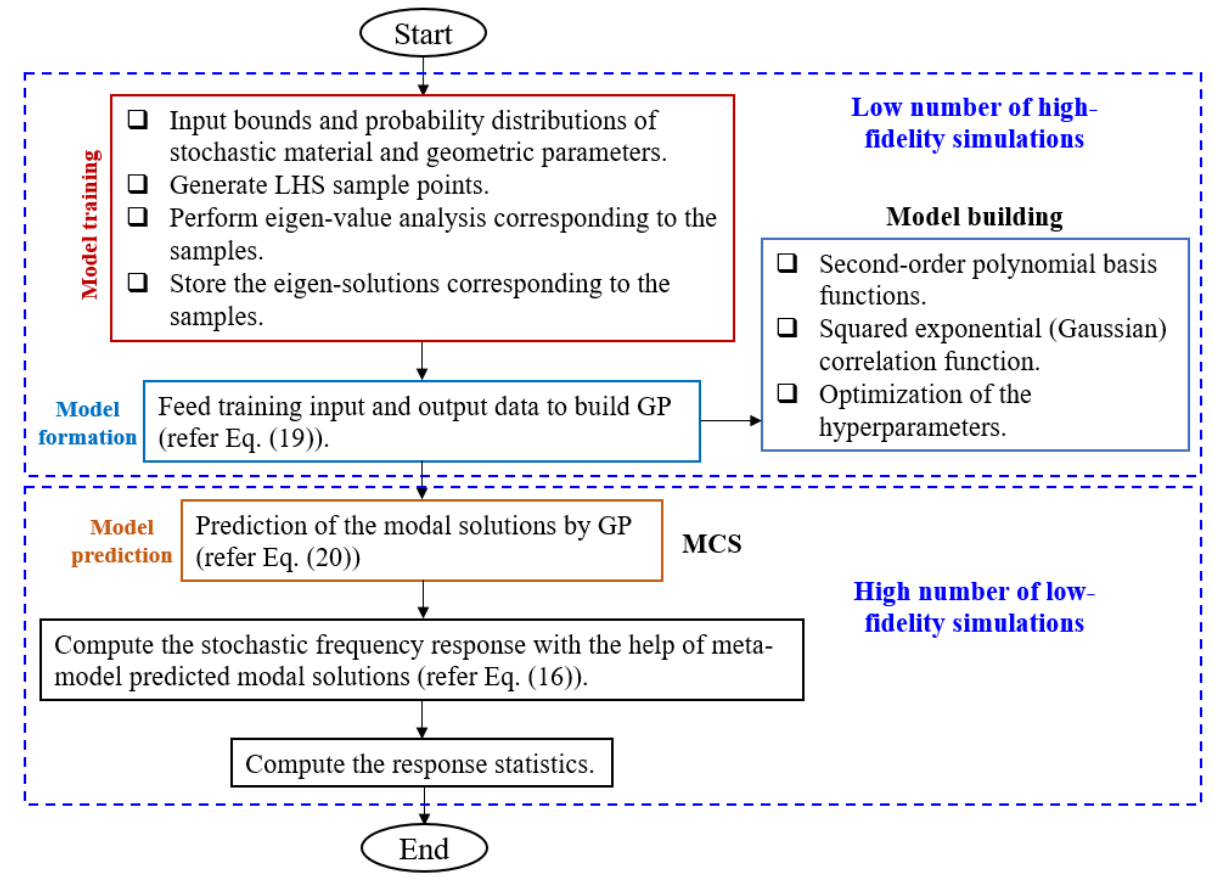

Figure 2: Flowchart of the proposed computational framework.

\section{Dynamic analysis of near-periodic structures}

The performance of the proposed approach is assessed by solving two problems in this section. The stochastic dynamic behaviour of systems having a repetitive geometrical configuration is studied. This is relevant as investigating the vibration characteristics of these periodic structures in the presence of uncertainties is critical due to their industrial applications in vibration attenuation and energy harvesting. In doing so, our main focus has been to accurately capture the stochastic variation in the dynamic response in a cost-effective manner due to material and geometric uncertainties (simulating the manufacturing variability). Complex scenarios, such as high levels of input uncertainties and approximation of closely spaced modes, have been undertaken individually.

\subsection{Mechanical models}

In this section, we have analyzed two common mechanical models, namely an elastically connected mass-springdamper chain system and a multiple beam system with attached masses connected by an elastic medium. The massspring-damper chain system, as shown in Fig. 3, consists of a finite number of masses with elastic connections. The elastic connection model is composed of a linear spring of stiffness $k_{i}$ and damper with coefficient $b_{i}$. In this study, we adopt the chain model with free boundary conditions, i.e. the first, $M_{1}$, and last, $M_{m}$, masses in the system are not connected to fixed boundaries. In the following section, we present the mathematical model corresponding to the specified boundary conditions analytically.

The second example consists of a multiple beam system (MBS) embedded in an elastic medium with attached point masses, as shown in Fig. 4. The mechanical model is composed of a set of $m$ isotropic elastic beams with elastic medium placed in between the beams. It is assumed that all beams have the same material and 


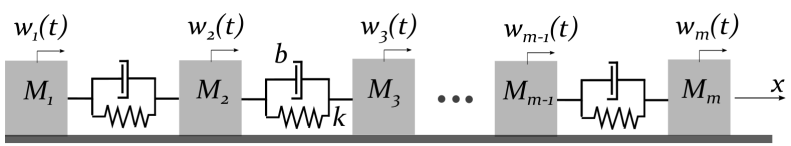

Figure 3: Mechanical model of the Damped Chain System (Example 1).

geometrical parameters, such as uniform cross-section area $A$, thickness $h$, length $L$, width $b$, elastic modulus $E$, Poisson's ratio $\nu$, mass density $\rho$. Moreover, we consider that the material of the elastic matrix, which is located between beams, is described by continuously distributed linear elastic springs and dampers with the elastic medium coefficients $\left(k_{i}, b_{i}\right), i=1,2,3, \ldots, m-1$. In general, we may consider different types of medium between the beams, such as viscoelastic or Pasternak models. Each beam has the same boundary conditions, and the same number of attached point masses, as shown in Fig. 4 . The transverse displacement of the $i$-th beam is denoted by $w_{i}(x, t),(i=1$, $2,3, \ldots, m)$. This analysis is limited to the case of Euler-Bernoulli beam theory, where the shear coefficient is neglected. We consider the case where the MBS is coupled in the "Free-Chain" configuration, i.e. the first and last beams in the system are not coupled to a fixed base, so that $k_{0}=b_{0}=0$ and $k_{m}=b_{m}=0$.

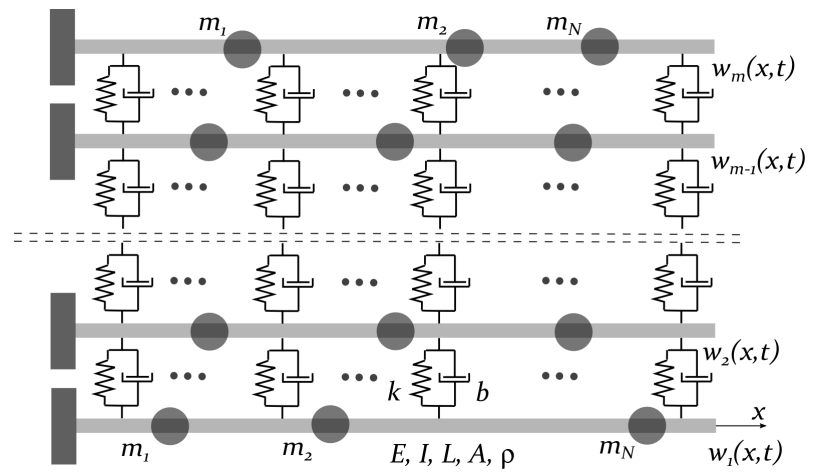

Figure 4: Mechanical model of the Multiple Beam System (Example 2).

\subsection{Analytical model - Example 1}

Introducing D'Alembert's principle, the following equations of motion of the mass-spring-damper systems can be formulated for the $i$-th mass of the system:

$$
\begin{aligned}
& M_{i} \ddot{w}_{i}+k_{i}\left(w_{i}-w_{i+1}\right)+k_{i-1}\left(w_{i}-w_{i-1}\right) \\
& +b_{i}\left(\dot{w}_{i}-\dot{w}_{i+1}\right)+b_{i-1}\left(\dot{w}_{i}-\dot{w}_{i-1}\right)=0
\end{aligned}
$$

where $w_{i}(t)$ is the displacement of the $i$-th mass of the system $m_{i}$, and $k_{i}$ and $b_{i}$ are the stiffness and damping parameters of the $i$-th spring and damper.

For the adopted "Free-Chain" system configuration of the elastically connected mass, the differential equations of motion in Eq.(22) can be reduced to

$$
M_{1} \ddot{w}_{1}+k_{1}\left(w_{1}-w_{2}\right)+b_{1}\left(\dot{w}_{1}-\dot{w}_{2}\right)=0
$$




$$
\begin{gathered}
M_{i} \ddot{w}_{i}+k_{i}\left(w_{i}-w_{i+1}\right)+k_{i-1}\left(w_{i}-w_{i-1}\right) \\
+b_{i}\left(\dot{w}_{i}-\dot{w}_{i+1}\right)+b_{i-1}\left(\dot{w}_{i}-\dot{w}_{i-1}\right)=0, \\
M_{m} \ddot{w}_{m}+k_{m-1}\left(w_{m}-w_{m-1}\right)+b_{m-1}\left(\dot{w}_{m}-\dot{w}_{m-1}\right)=0,
\end{gathered}
$$

The corresponding boundary conditions for the system in Fig. 3 give,

$$
i=0: \quad k_{0}=b_{0}=0, \quad \text { and } \quad i=m: \quad k_{m}=b_{m}=0 .
$$

To determine an analytical solution for the damping ratio and damped natural frequency for multiple connected mass-spring-damper systems, the first step is to reduce the system of differential equations Eq.(22) to a system of algebraic equations by assuming a solution of the form of $w_{i}(t)=W_{i} e^{\mathrm{i} \omega t}$, where $\mathrm{i}=\sqrt{-1}$ is the unit imaginary. The resulting system of algebraic equations then determine the characteristic equation, and the solution is obtained by assuming the $i$-th algebraic equation has the form $W_{i}=N \cos \left(i \phi_{s}\right)+P \sin \left(i \phi_{s}\right)$, as shown in [3]. The parameter $\phi_{s}$ depends on the boundary conditions of the chain system, and for the "Free-Chain" system is determined as $\phi_{s}=\frac{s \pi}{m}$, where $s=0,1,2, \ldots, m-1$ represents the mode number. The general form of the complex natural frequency is

$$
\omega_{s}=\mathrm{i} \frac{b}{m}\left(1-\cos \phi_{s}\right) \pm \sqrt{2 \frac{k}{m}\left(1-\cos \phi_{s}\right)-\frac{b^{2}}{m^{2}}\left(1-\cos \phi_{s}\right)^{2}}
$$

The real part of the complex natural frequency corresponds to the damped natural frequency, and the imaginary part is related to the damping ratio. The natural frequency and damping ratio are then

$$
\begin{gathered}
\Omega_{s}=\sqrt{2 \frac{k}{m}\left(1-\cos \phi_{s}\right)-\frac{b^{2}}{m^{2}}\left(1-\cos \phi_{s}\right)^{2}}, \\
\zeta_{s}=\frac{b}{m}\left(1-\cos \phi_{s}\right) .
\end{gathered}
$$

\subsection{Analytical model - Example 2}

In order to derive the governing equations of motion of the multiple beam system (MBS) with added mass, Hamilton's principle and Euler-Bernoulli beam theory are used to give the following equation of motion for the $i$-th beam

$$
\begin{gathered}
E I w_{i}^{\prime \prime \prime \prime}+\left(\rho A+\sum_{p=1}^{N} m_{p i} \delta\left(x-a_{p(i)}\right)\right) \ddot{w}_{i}+k_{i}\left(w_{i}-w_{i+1}\right) \\
+k_{i-1}\left(w_{i}-w_{i-1}\right)+b_{i}\left(\dot{w}_{i}-\dot{w}_{i+1}\right)+b_{i-1}\left(\dot{w}_{i}-\dot{w}_{i-1}\right)=q_{i}(x, t) \\
\text { for } \quad i=1,2, \ldots, m-1, m
\end{gathered}
$$

where $w_{i}=w_{i}(x, t)$ is the transverse displacement of the $i$-th beam, and $I, \rho$ and $A$ are the cross-sectional moment of inertia, the material mass density and the cross-sectional area of the $i$-th beam, respectively. $x \in[0, L]$ is the axial coordinate, $q_{i}(x, t)$ is an arbitrary transverse load acting on the $i$-th beam, $m_{p i}$ is the $p$-th mass attached to the $i$-th beam at $a_{p(i)}$, and $\delta$ is the Dirac function. $(\bullet)^{\prime} \operatorname{denotes} \partial(\bullet) / \partial x$, and $(\dot{\bullet})$ denotes $\partial(\bullet) / \partial t$. 
The corresponding boundary conditions are

$$
\begin{gathered}
x=0: \quad w_{i}(0, t)=0, \quad w_{i}^{\prime}(0, t)=0, \\
x=L: \quad E I w_{i}^{\prime \prime}(L, t)=0, \quad E I w_{i}^{\prime \prime \prime}(L, t)=0,
\end{gathered}
$$

From Eq. (30), the "Free-Chain" system configuration of the MBS, as shown in Fig. 4, can be obtained as

$$
\begin{gathered}
E I w_{1}^{\prime \prime \prime \prime}+\left(\rho A+\sum_{p=1}^{N} m_{p 1} \delta\left(x-a_{p(1)}\right)\right) \ddot{w}_{1}+k_{1}\left(w_{1}-w_{2}\right) \\
+b_{1}\left(\dot{w}_{1}-\dot{w}_{2}\right)=q_{1}(x, t), \quad i=1 \\
E I w_{i}^{\prime \prime \prime \prime}+\left(\rho A+\sum_{p=1}^{N} m_{p i} \delta\left(x-a_{p(i)}\right)\right) \ddot{w}_{i}+k_{i}\left(w_{i}-w_{i+1}\right) \\
+k_{i-1}\left(w_{i}-w_{i-1}\right)+b_{i}\left(\dot{w}_{i}-\dot{w}_{i+1}\right)+b_{i-1}\left(\dot{w}_{i}-\dot{w}_{i-1}\right)=q_{i}(x, t), \\
\text { for } i=2, \ldots, m-1 \\
E I w_{m}^{\prime \prime \prime \prime}+\left(\rho A+\sum_{p=1}^{N} m_{p m} \delta\left(x-a_{p(m)}\right)\right) \ddot{w}_{m}+k_{m-1}\left(w_{m}-w_{m-1}\right) \\
+b_{m-1}\left(\dot{w}_{m}-\dot{w}_{m-1}\right)=q_{m}(x, t), i=m
\end{gathered}
$$

In the following, we have used FE modelling to discretize the system [65] and the free vibration problem is solved as

$$
\left(\mathbf{K}-\omega^{2} \mathbf{M}\right) \Delta=\mathbf{0}
$$

where $\mathbf{K}$ and $\mathbf{M}$ are the global stiffness and mass matrices of the MBS, respectively. The resulting matrices from the FE formulation are provided in Appendix 1.

\subsection{Stochastic modelling}

After describing the deterministic models of the near-periodic structures, the stochastic modelling is briefly highlighted in this section to illustrate how the proposed computational framework (Section 3) is employed to solve the examples (Section 4.1-4.3) in the next section (Section 5). The mass and stiffness terms of each DOF in the analytical model of the damped chain system (example 1) have been considered to be random. The material and geometric parameters at the finite element level of the multiple beam mass system considered in example 2 have been assumed as random. Thus, in both examples, the input uncertainty is propagated to the global mass and stiffness matrices, and a stochastic eigenvalue problem is posed taking into account the global random system matrices for the undamped case. Specifically, each of the modal solutions is represented by an individual GP model and they are estimated using Eq. (20). Corresponding to a random realization of the modal solutions, Eq.(16) is used to obtain the dynamic response in the frequency domain. Consequently, the propagation of the input uncertainty to the dynamic response using the GP only requires a nominal number of analytical or FE simulations 
of the actual system (Fig. 2). The variation of the natural frequencies and frequency response functions are studied in Section 5 . The results are validated by dMCS.

Note that instead of GP, any meta-modelling technique can be used in the above non-intrusive stochastic framework, provided it is capable of capturing the non-linear response variation due to the high level of uncertainty and the presence of repeated or close modes.

\section{Numerical study}

\subsection{Results and discussion: Example 1}

Ten DOFs have been considered to undertake the numerical investigation of example 1 . The nominal parameter values used are $M_{i}=1, k_{i}=10$ and $b_{i}=0.01$. For incorporating randomness in the system, the mass and stiffness of each DOF are assumed to be lognormally distributed with $5 \%$ uncertainty. As the damping matrix $\mathbf{C}$ is assumed to be proportional and thus derived from the stochastic mass and stiffness matrices, in a sense $\mathbf{C}$ is also random in the analysis. In total, there are thirty stochastic variables considered in the analysis. Thirty samples were generated by a Latin hypercube sampling (LHS) scheme [66] to train the GP meta-model. This was implemented using the "lhsdesign" built-in MATLAB toolbox and the "maximin" option which maximises the minimum distance between points. The DACE platform was employed to implement the GP model in this work [58]. A Gaussian correlation function was assumed to construct the GP. The GP meta-model was constructed using thirty actual function evaluations corresponding to the input sample points. For validation of the GP predicted frequency responses, $10^{4}$ samples of dMCS were generated.

Since only thirty samples for the GP were adequate to approximate the stochastic dynamic response, an error convergence study to vary the number of samples has not been performed for this example. The variation in each of the natural frequencies is shown in Fig. 5. The variation in the natural frequencies from the actual discrete chain model (Fig. 5a) and the GP based prediction (Fig. 5b) are compared. The similarity of Figs. 5a and 5b demonstrates that good approximation accuracy has been obtained by the GP. An interesting point in terms of the input uncertainty propagation to the eigensolution space, shown by Fig. 5, is that the higher frequencies are characterized with higher variation compared to the lower frequencies. This is a positive indication as it is evident that lower natural frequencies play a more dominant role in frequency responses than the higher frequencies. Thus, it can be expected that the frequency response in the low-frequency region will have lower stochastic variation. Similar observations can be seen from the FRF band plots in Fig. 6 where the band in the low-frequency region up to $2 \mathrm{rad} / \mathrm{s}$ is narrower compared to that in the higher frequency region.

The complete FRF matrix obtained by GP is observed to be superimposed with that of MCS, illustrating satisfactory approximation accuracy. A sample FRF band plot is presented in Fig. 6. To further substantiate the accuracy achieved by the GP meta-model in Fig. 6 , the relative $L_{2}$ error in the coefficient of variation (C.O.V. = Standard deviation/Mean) of $\mathrm{FRF} \mathrm{H}(2,2)$ is presented in Fig. 7. It can be observed that the error is well below $10^{-5}$ for all values of forcing frequency. The variation in the natural frequencies in Fig. 5 and the frequency response 


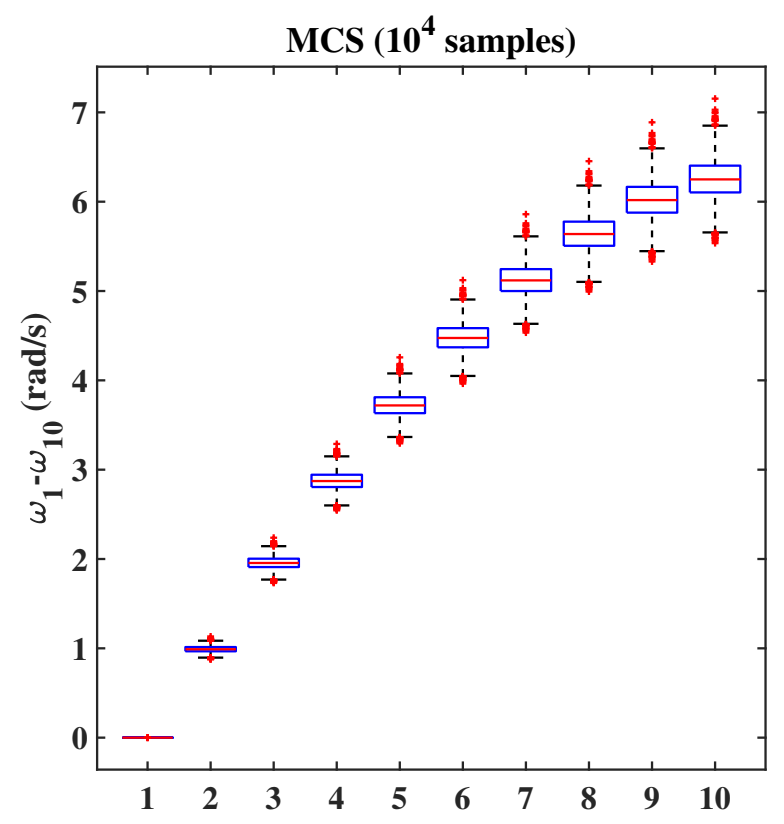

(a) $\operatorname{MCS}\left(10^{4}\right.$ samples)

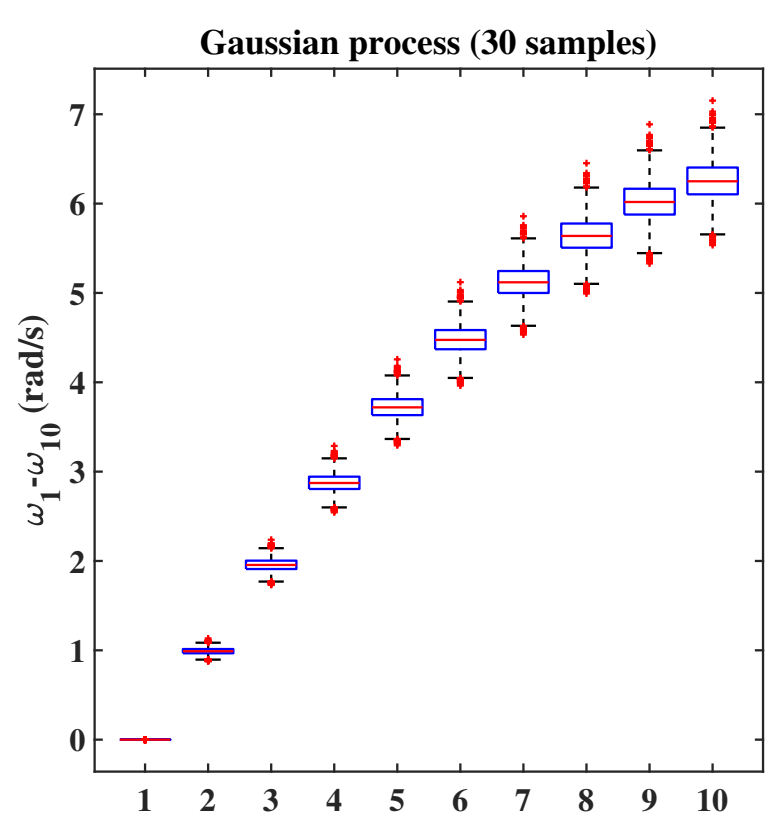

(b) Gaussian process (30 samples)

Figure 5: Box plots to illustrate variation of the natural frequencies by using (a) MCS (10 ${ }^{4}$ samples) and (b) Gaussian Process (30 samples). The ten natural frequencies of the system are given along the $\mathrm{x}$-axis and the $\mathrm{y}$-axis gives the corresponding estimated natural frequencies. The central mark (horizontal red line) in each box indicates the median, and the top and bottom edges of the box indicate the 75 th and 25th percentiles, respectively. The whiskers (in black) are the most extreme data points and the outliers are denoted using the '+' symbol in red.

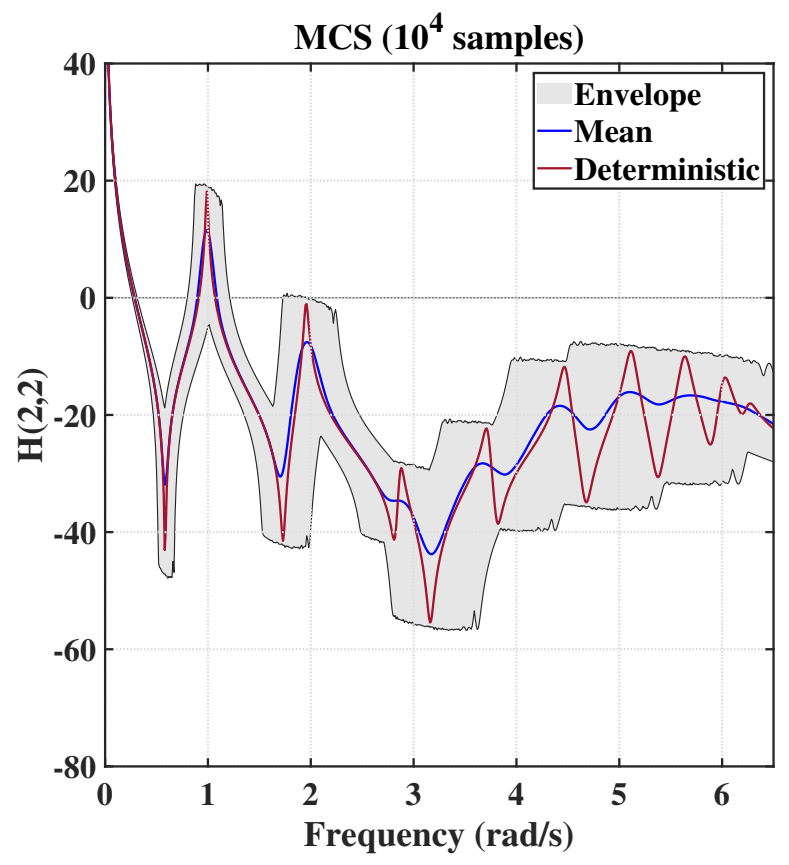

(a) $\operatorname{MCS}\left(10^{4}\right.$ samples $)$

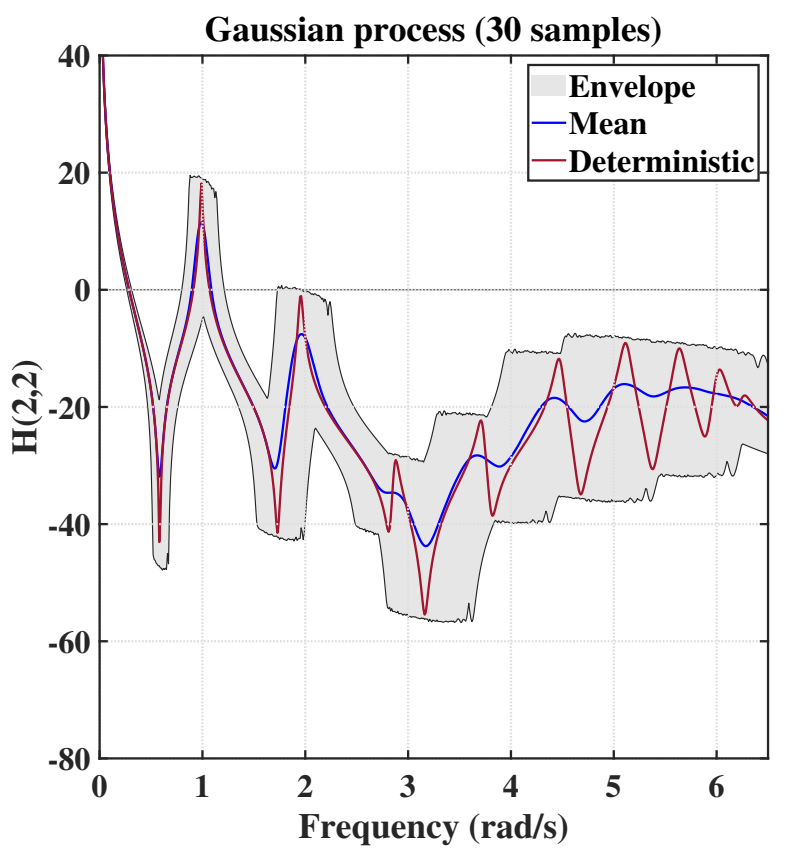

(b) Gaussian process (30 samples)

Figure 6: Transverse displacement frequency response function (FRF) band plots (dB) by using (a) MCS (10 $0^{4}$ samples) and (b) Gaussian Process (30 samples). The FRF $\mathrm{H}(2,2)$ is shown along the $\mathrm{y}$-axis with varying forcing frequency values on the $\mathrm{x}$-axis. The deterministic FRF plot (in blue) was evaluated at the nominal (mean) values of the random input parameters. The mean FRF plot (in red) is the average of the FRF corresponding to the random realizations over each forcing frequency. 
in Fig. 6 illustrate the effect of $5 \%$ variation in the input parameters and highlight the necessity of performing stochastic response analysis.

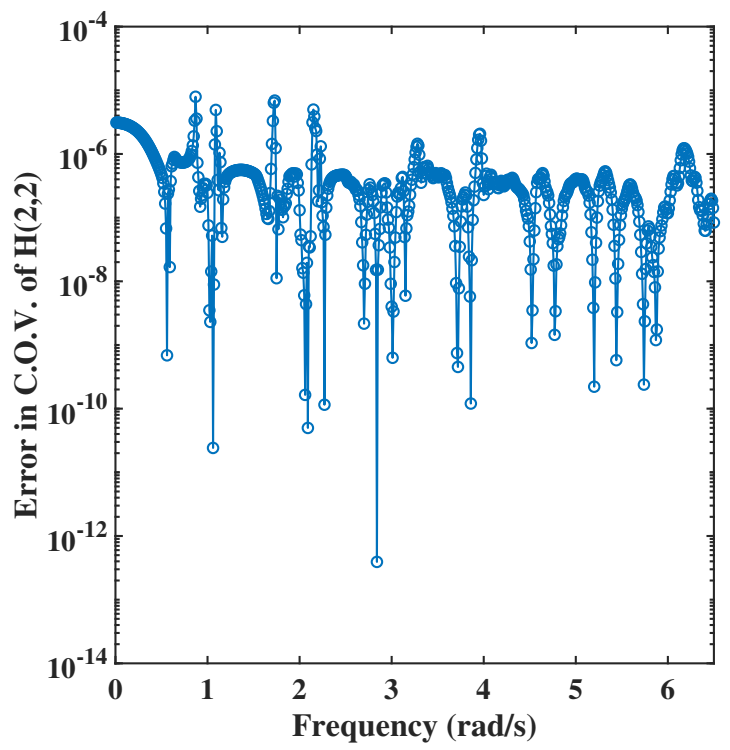

Figure 7: Relative $L_{2}$ error in the coefficient of variation (C.O.V) of the response FRF H(2,2). C.O.V is obtained as the ratio of the standard deviation to the mean of the FRF. The relative $L_{2}$ error obtained by GP (30 samples) has been computed w.r.t MCS (10 4 samples). The error is reported for every value of forcing frequency to show the performance of the GP and its approximation potential to estimate the complete dynamic behaviour.

To further access the accuracy of the GP model, the level of uncertainty in the input parameters is increased. Figure 8 shows the relative $L_{2}$ error in estimating the natural frequencies by using GP (30 samples) in the presence of $10 \%$ and $20 \%$ variations in the input parameters. It can be observed from both the cases (Figs. 8a and 8b) that acceptable error values in the range of $10^{-4}-10^{-8}$ are obtained for all of the natural frequencies, illustrating the robust potential of the GP meta-model in dealing with high levels of uncertainty. This is an advantage of the GP meta-model compared to perturbation based approaches which often fail to capture the response variation due to high input fluctuations.

\subsection{Results and discussion: Example 2}

The stochastic frequency response of the multiple beam system with added masses (Example 2) is presented in this sub-section. Three beams with three attached masses were considered in the numerical study. The description of all of the random input parameters is provided in Table 1. The length of the beams was $0.8 \mathrm{~m}$ and all of the damping ratios were $\xi_{i}=0.01$. Table 1 shows that six material and geometric parameters are considered as random in each finite element. Each of the beams is discretized into twenty finite elements.

The results of the stochastic response analysis are now discussed. To calculate the results, the sampling scheme and the correlation function in the GP are the same as Example 1. To determine the optimal number of samples required to build the meta-model, the convergence of the relative $L_{2}$ error in approximating the mean of the natural frequencies is studied. Figure 9 shows the error convergence of the mean of the first six natural frequencies 


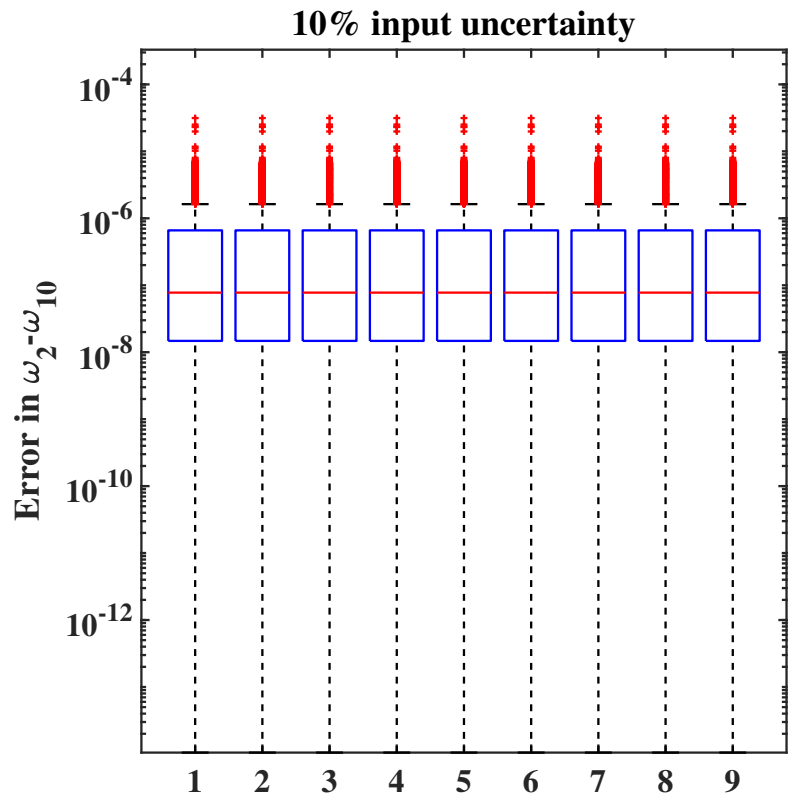

(a) $10 \%$ input uncertainty

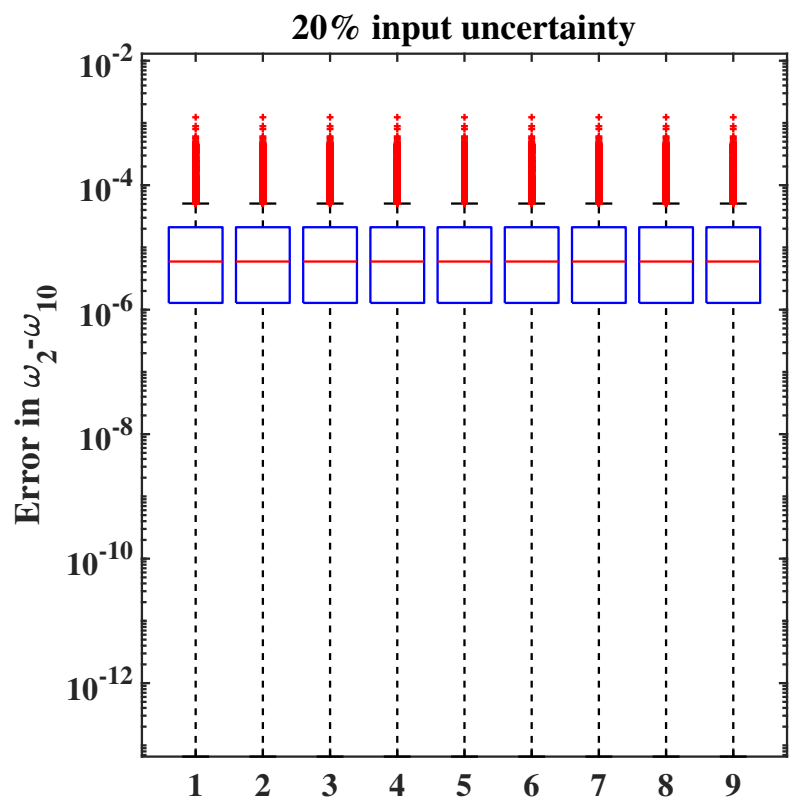

(b) $20 \%$ input uncertainty

Figure 8: Relative $L_{2}$ error in approximating the natural frequencies by GP (30 samples) w.r.t. MCS ( $10^{4}$ samples) in the presence of (a) $10 \%$ (b) $20 \%$ uncertainty in the input parameters. The low range of error values obtained $\left(10^{-4}-10^{-8}\right)$ illustrate the robust approximation capability of the GP for high levels of uncertainty. The central mark (horizontal red line) in each box indicates the median, the top and bottom edges of the box indicate the 75th and 25th percentiles, respectively. The whiskers (in black) are the most extreme data points and the outliers are denoted using the ' + ' symbol in red.

Table 1: Description of the random input parameters in Example 2

\begin{tabular}{lllll}
\hline Variables & Unit & Distribution & Mean & C.O.V. \\
\hline Elastic modulus & $\mathrm{N} / \mathrm{m}^{2}$ & Lognormal & $3.2 \times 10^{9}$ & 0.1 \\
Density & $\mathrm{kg} / \mathrm{m}^{3}$ & Lognormal & 1190 & 0.1 \\
Poisson's ratio & $/$ & Lognormal & 0.25 & 0.05 \\
C/S height & $\mathrm{m}$ & Lognormal & 0.003 & 0.05 \\
C/S width & $\mathrm{m}$ & Lognormal & 0.02 & 0.05 \\
Layer stiffness & $\mathrm{N} / \mathrm{m}^{2}$ & Lognormal & 100 & 0.1 \\
\hline
\end{tabular}

estimated by the GP with varying numbers of sample points. Based on Fig. 9, forty-five samples were used to train the GP model as the error is below or close to $10^{-4}$ for all of the frequencies. Thus, the GP is built using forty-five actual function evaluations corresponding to the input sample points. The variation in the first fifteen natural frequencies are given in Fig. 10. The variation in the natural frequencies from the actual FE model (Fig. 10a) and the GP based prediction (Fig. 10b) are compared. The similarity of Figs. 10a and 10b demonstrate that good approximation accuracy has been obtained by the GP.

Figure 10 shows that the system is characterized by sets of close modes. The robust approximation capability demonstrated by the GP with a nominal number of samples is of special interest here, as capturing the variation in the modal space in systems having close modes is difficult due to mode degeneration phenomena (including mode veering, mode crossing and coalescence) [67]. This is one of the key highlights of the work as (i) it is difficult to distinguish between neighbouring modes based on their eigenvalues due to mode crossing and (ii) for mode veering, 


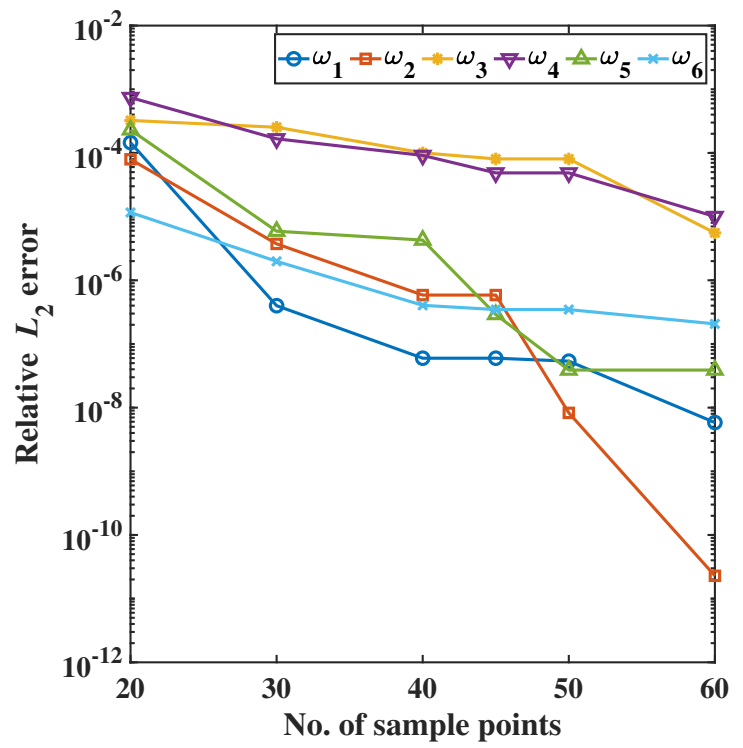

Figure 9: Convergence of relative $L_{2}$ error of the first six mean natural frequencies of the multiple beam mass system estimated by the Gaussian Process model w.r.t MCS ( $10^{4}$ samples) for varying number of sample (training) points. The optimal number of sample points required to train the GP model is determined to achieve satisfactory accuracy level.

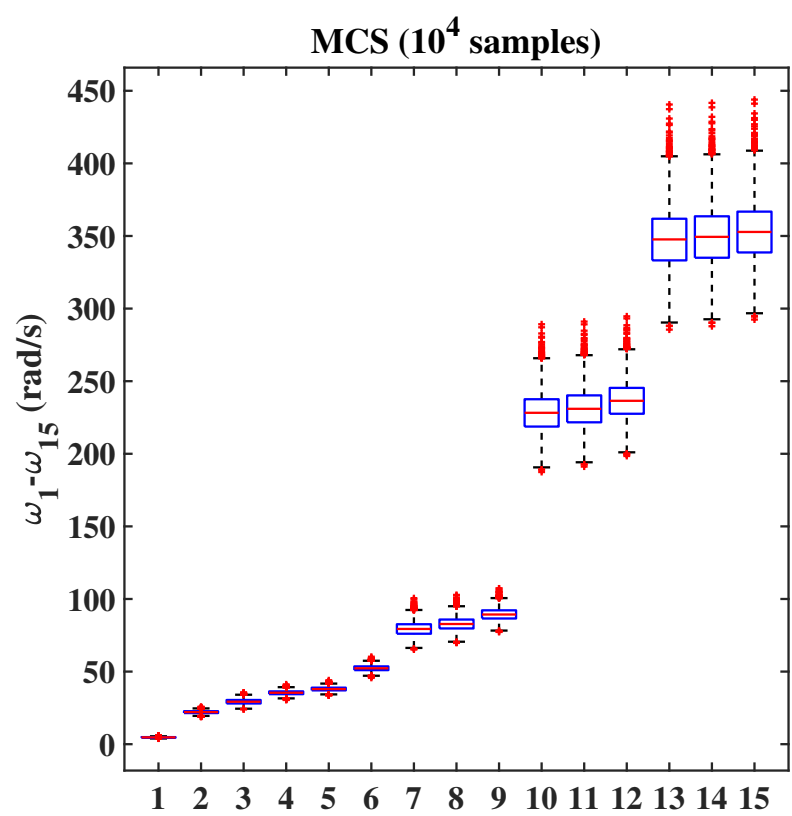

(a) $\operatorname{MCS}\left(10^{4}\right.$ samples $)$

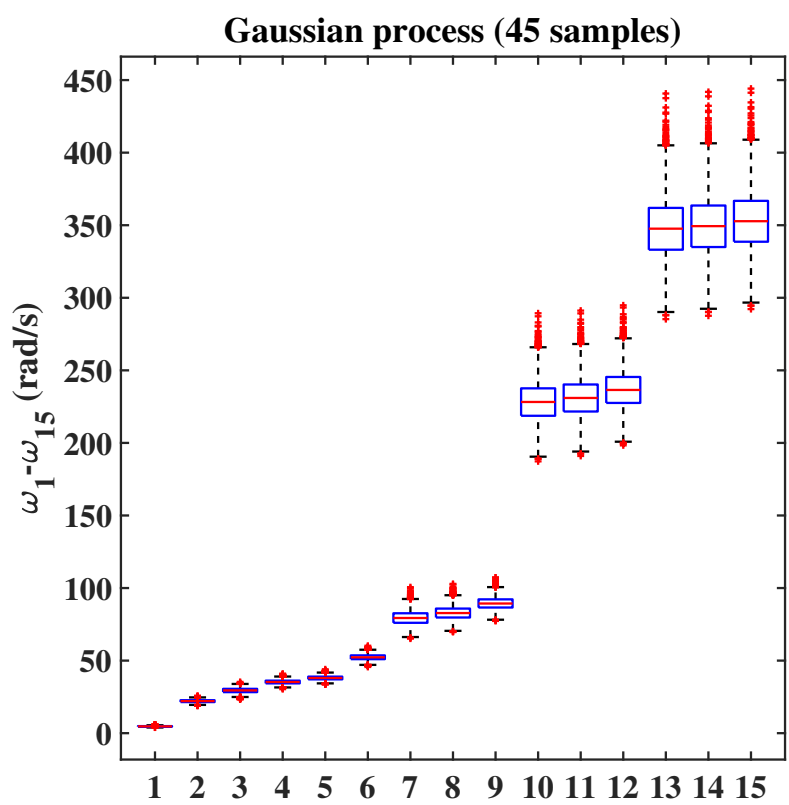

(b) Gaussian process (45 samples)

Figure 10: Box plots to illustrate variation of the first fifteen natural frequencies of the multiple beam mass system by using (a) MCS (10 4 samples) and (b) Gaussian Pprocess (45 samples). The first fifteen natural frequencies of the system are shown along the y-axis and the $\mathrm{x}-$ axis gives the estimated natural frequencies. The central mark (horizontal red line) in each box indicates the median, the top and bottom edges of the box indicate the 75 th and 25 th percentiles, respectively. The whiskers (in black) are the most extreme data points and the outliers are denoted using the '+' symbol in red.

even small input perturbations may lead in a relatively large variation of the eigenvectors. Thus, tracking the non-linear evolution of the modal solutions in the above cases may prove to be difficult and can easily lead to inaccurate predictions by the meta-model [25]. As observed in the previous example, the higher natural frequencies 
in the MBS are characterized with higher variation compared to the lower frequencies (Fig. 10). This is a positive indication as the lower natural frequencies play a more dominant role in the frequency responses than the higher frequencies.

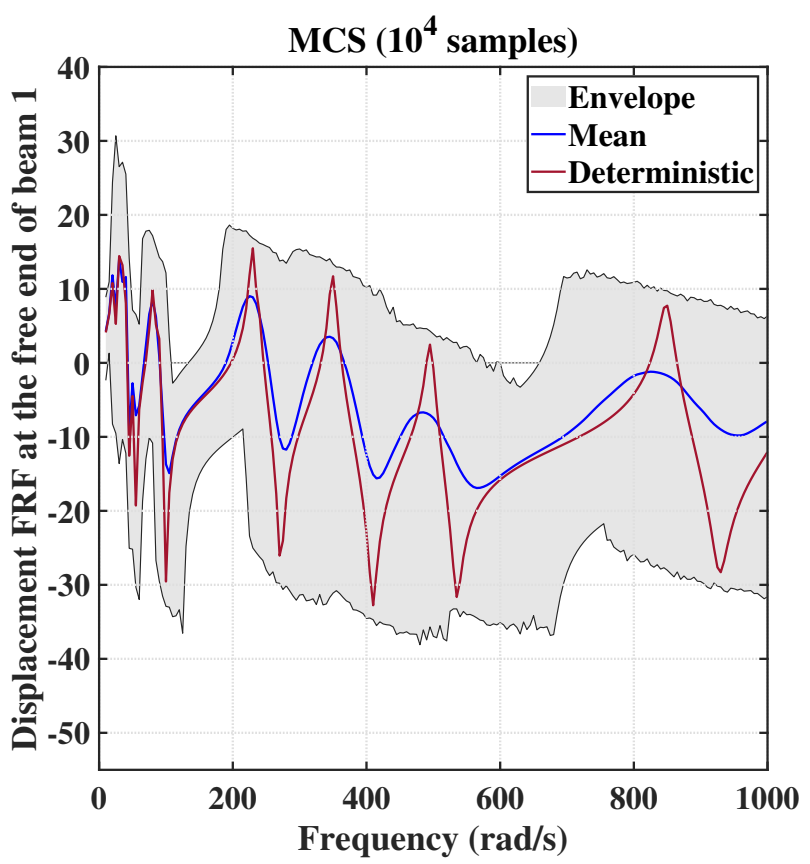

(a) MCS (10 samples)

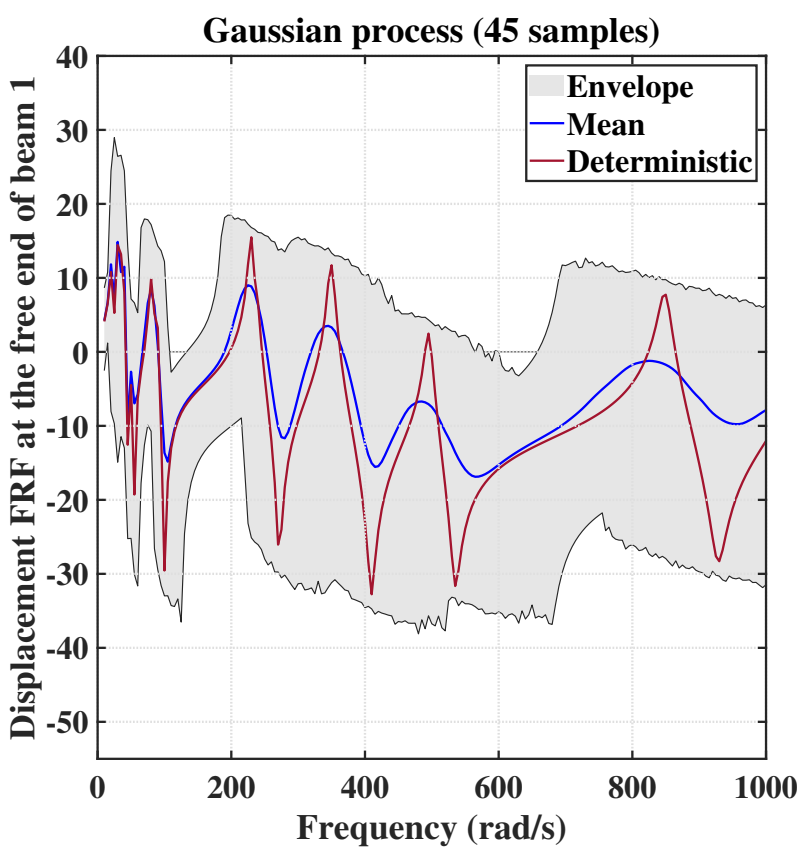

(b) Gaussian process (45 samples)

Figure 11: Transverse displacement frequency response function band plots (dB) by using (a) MCS (10 ${ }^{4}$ samples) and (b) Gaussian Process (45 samples). The direct displacement FRF term at the free end of the top beam is shown for varying forcing frequencies. The deterministic FRF plot (in blue) was evaluated at the nominal (mean) values of the random input parameters. The mean FRF plot (in red) is the average of the FRF corresponding to the random realizations for each forcing frequency.

The complete FRF matrix obtained by the GP is observed to be superimposed with that of MCS, illustrating satisfactory approximation accuracy. A sample FRF band plot showing the variation of the transverse displacement at the free end of the top beam is presented in Fig. 11. The similarity of the results obtained by the GP (Fig. 11b) with those of MCS (Fig. 11a) show that the former achieves a satisfactory level of accuracy in estimating the response of the multiple beam mass system with limited computational effort. The deterministic response (i.e. the response obtained corresponding to the mean values of input random parameters) is also presented to highlight its difference to the mean response and hence illustrating the need to consider uncertainties during dynamic analysis. To further substantiate the accuracy achieved by the GP meta-model in Fig. 11, the relative $L_{2}$ error in the coefficient of variation (C.O.V. = Standard deviation/Mean) of the FRF at the free end of the top beam is presented in Fig. 12. The error is approximately $10^{-5}$ for most values of forcing frequency. The error is slightly higher (in the range $10^{-2}-10^{-3}$ ) at frequencies close to zero due to rigid body modes. The variation in the natural frequencies in Fig. 10 and the frequency response in Fig. 11 illustrate the effect of variation in the input parameters (shown in Table 1) and highlight the necessity of performing stochastic response analysis.

An important point to note is that to ensure reasonable approximation accuracy by the meta-model, the sign 


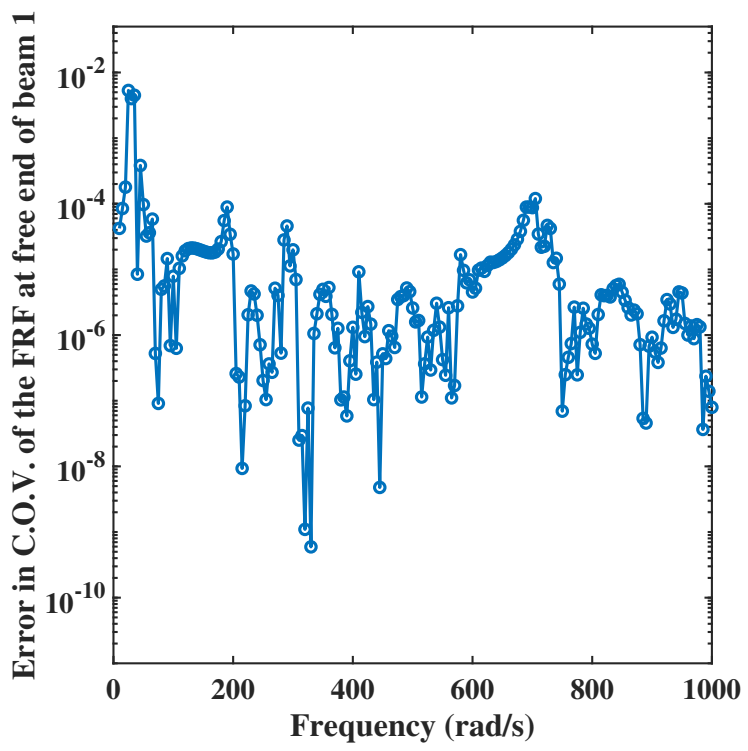

Figure 12: Relative $L_{2}$ error in the coefficient of variation (C.O.V) of the response direct displacement FRF term at the free end of the top beam. C.O.V is obtained as the ratio of the standard deviation to the mean of the FRF. The relative $L_{2}$ error obtained by th eGP (45 samples) has been computed w.r.t MCS ( $10^{4}$ samples). The error is reported for every forcing frequency to show the performance of the GP and its approximation potential to estimate the complete dynamic behaviour.

of the modes was kept consistent with a reference mode shape.

\section{Conclusions}

The contribution of the study lies in the fact that a rigorous investigation of the stochastic dynamics of near periodic meta-structures has been presented. It has been observed that the natural frequencies and the dynamic response have significant variation due to manufacturing anomalies (simulated by perturbing the material and geometric parameters of the structural models). This highlights the importance of accounting for uncertainties while analyzing periodic structures prone to inevitable manufacturing fluctuations and eventually to prevent deterioration of their performance.

To address large-scale FE models of these repetitive systems in the presence of uncertainties, we propose a GP based framework to significantly reduce the computational effort and at the same time estimate the dynamic response with satisfactory accuracy. In doing so, the input uncertainties are mapped onto the eigenspace instead of directly computing the frequency response. By approximating the eigensolutions, the need to perform high-fidelity simulations for every forcing frequency can be avoided. Moreover, as eigenvalue analysis is essentially a linear problem, the input-output relation can be easily mapped with a conventional meta-model with a nominal number of sample points. This has been illustrated by the successful implementation of the plain vanilla version of GP while solving the undertaken examples in this study.

It is worth noting that the trained GP model, which is essentially a physics informed data-driven model, is inherently capable of predicting the dynamic behaviour corresponding to a new set of system parameters within their pre-defined bound. Therefore, the same methodology can be used as a type of transfer learning model to 
obtain the dynamics of similar systems having insufficient information or missing data sets, without having to re-train the complete model from scratch.

The results of the first example demonstrated that the GP proved to be robust enough to capture the natural frequencies (well separated) even for a high level of input uncertainty. A key highlight based on the results of the second example was the excellent performance of the GP in tracking the non-linear evolution of the dynamic response in the presence of close modes. This is of special interest as it is well known that capturing the variation of the modal space in systems having close modes is difficult due to mode degeneration phenomena (including mode veering, mode crossing and coalescence). Therefore, the work illustrates a generalized meta-modelling scheme for systems having close or repeated modes. Both of the above cases are commonly encountered in periodic structures and therefore, the results achieved will be useful for further investigation because very few works exist on meta-modelling of systems with close modes.

The proposed framework is simple and general in the sense that any FE model and meta-modelling technique can be seamlessly incorporated. The study is one of the first to develop a stochastic computational framework to explore the dynamic behaviour of meta-structures. The study is expected to attract attention to efficiently investigate the stochastic dynamic behaviour of periodic structures, which are popular for their vibration attenuation and energy harvesting capabilities. Another potential application of the proposed approach is for computationally expensive reliability-based and/or robust design optimization frameworks for stochastic dynamic systems, where UQ is coupled within the optimization loop and involves a significantly high number of high-fidelity simulations. The proposed approximation strategy can be utilized to reduce the computational effort.

We are currently looking at a similar type of model which is representative of periodic structures and study the effect of uncertainties on the efficiency of vibration mitigation. To further improve the computational effort of the present framework, concepts of model-order reduction are being investigated.

\section{Acknowledgements}

Dr. D. Karličić and Prof. S. Adhikari were supported by the Marie SkÅĆodowska-Curie Actions - European Commission: 799201-METACTIVE. Dr. T. Chatterjee and Prof. M. I. Friswell gratefully acknowledge the support of the Engineering and Physical Sciences Research Council through the award of a Programme Grant "Digital Twins for Improved Dynamic Design", grant number EP/R006768.

[1] H. Deng, Y. Du, Z. Wang, J. Ye, J. Zhang, M. Ma, X. Zhong, Poly-stable energy harvesting based on synergetic multistable vibration, Communications Physics 2 (1) (2019) 1-10.

[2] S. Paunović, M. Cajić, D. Karličić, M. Mijalković, Dynamics of fractional-order multi-beam mass system excited by base motion, Applied Mathematical Modelling 80 (2020) 702-723. 
[3] D. Karličić, P. Kozić, R. Pavlović, Nonlocal vibration and stability of a multiple-nanobeam system coupled by the winkler elastic medium, Applied Mathematical Modelling 40 (2) (2016) 1599-1614.

[4] Z. C. He, J. Y. Hu, E. Li, An uncertainty model of acoustic metamaterials with random parameters, Computational Mechanics 62 (2018) 1023-1036.

[5] J. Henneberg, J. S. Gomez Nieto, K. Sepahvand, A. Gerlach, H. Cebulla, S. Marburg, Periodically arranged acoustic metamaterial in industrial applications: The need for uncertainty quantification, Applied Acoustics $157(2020) 107026$.

[6] P. Celli, B. Yousefzadeh, C. Daraio, S. Gonella, Bandgap widening by disorder in rainbow metamaterials, Applied Physics Letters 114 (2019) 091903.

[7] L. Hinke, F. Dohnal, B. R. Mace, T. Waters, N. Ferguson, Component mode synthesis as a framework for uncertainty analysis, Journal of Sound and Vibration 324 (2009) 161-178.

[8] H. A. Babaa, S. Nandi, T. Singh, M. Nouh, Uncertainty Quantification of Tunable Elastic Metamaterials using Polynomial Chaos, Journal of Applied Physics 127 (2020) 015102.

[9] G. Schuëller, H. Jensen, Computational methods in optimization considering uncertainties âĂ An overview, Computer Methods in Applied Mechanics and Engineering 198 (1) (2008) 2-13.

[10] N. Vu-Bac, T. Lahmer, X. Zhuang, T. Nguyen-Thoi, T. Rabczuk, A software framework for probabilistic sensitivity analysis for computationally expensive models, Advances in Engineering Software 100 (2016) 1931.

[11] X. Du, W. Chen, Methodology for managing the effect of uncertainty in simulation-based design, AIAA Journal 38 (8) (2000) 1471-1478.

[12] G. Muscolino, G. Ricciardi, P. Cacciola, Monte carlo simulation in the stochastic analysis of non-linear systems under external stationary poisson white noise input, International Journal of Nonlinear Mechanics 38 (2003) $1269-1283$.

[13] V. Dubourg, B. Sudret, F. Deheeger, Metamodel-based importance sampling for structural reliability analysis, Probabilistic Engineering Mechanics 33 (2013) 47-57.

[14] V. Dubourg, B. Sudret, M. Bourinet, Reliability-based design optimization using kriging surrogates and subset simulation, Structural Multidisciplinary Optimization 44 (5) (2011) 673-690.

[15] M. Murugan, R. Ganguli, D. Harursampath, Surrogate based design optimisation of composite aerofoil crosssection for helicopter vibration reduction, The Aeronautical Journal 116 (1181) (2012) 709-725. 
[16] T. Chatterjee, S. Chakraborty, R. Chowdhury, A Critical Review of Surrogate Assisted Robust Design Optimization, Archives of Computational Methods in Engineering 26 (1) (2019) 245-274.

[17] T. Mukhopadhyay, S. Chakraborty, S. Dey, S. Adhikari, R. Chowdhury, A critical assessment of Kriging model variants for high-fidelity uncertainty quantification in dynamics of composite shells, Archives of Computational Methods in Engineering 24 (3) (2017) 495-518.

[18] S. Dey, T. Mukhopadhyay, S. Adhikari, Metamodel based high-fidelity stochastic analysis of composite laminates: A concise review with critical comparative assessment, Composite Structures 171 (2017) 227-250.

[19] B. Bhattacharyya, A Critical Appraisal of Design of Experiments for Uncertainty Quantification, Archives of Computational Methods in Engineering 0 (0). doi:10.1007/s11831-017-9211-x.

[20] J. Cheng, Z. Liu, Z. Wu, X. Li, J. Tan, Robust optimization of structural dynamic characteristics based on adaptive Kriging model and CNSGA, Structural and Multidisciplinary Optimization 51 (2) (2014) $423-437$.

[21] C. E. Rasmussen, C. K. I. Williams, Gaussian Processes for Machine Learning, The MIT Press, Cambridge, Massachusetts London, England, 2006.

[22] S. Chakraborty, R. Chowdhury, Graph-Theoretic-Approach-Assisted Gaussian Process for Nonlinear Stochastic Dynamic Analysis under Generalized Loading, Journal of Engineering Mechanics 145 (12) (2019) 1-16.

[23] T. Chatterjee, R. Chowdhury, An efficient sparse Bayesian learning framework for stochastic response analysis, Structural Safety 68 (2017) (2017) 1-14.

[24] M. Guo, J. S. Hesthaven, Reduced order modeling for nonlinear structural analysis using Gaussian process regression, Computer Methods in Applied Mechanics and Engineering 341 (2018) 807-826.

[25] J. Lu, Z. Zhan, D. W. Apley, W. Chen, Uncertainty propagation of frequency response functions using a multi-output Gaussian Process model, Computers and Structures 217 (2019) 1-17.

[26] H. Mohammadi, P. Challenor, M. Goodfellow, Emulating dynamic non-linear simulators using Gaussian processes, Computational Statistics and Data Analysis 139 (2019) 178-196.

[27] K. Worden, W. E. Becker, T. J. Rogers, E. J. Cross, On the confidence bounds of Gaussian process NARX models and their higher-order frequency response functions, Mechanical Systems and Signal Processing 104 (2018) 188-223.

[28] D. Mead, Wave propagation in continuous periodic structures: research contributions from southampton, 1964-1995, Journal of sound and vibration 190 (3) (1996) 495-524.

[29] D. Mead, A general theory of harmonic wave propagation in linear periodic systems with multiple coupling, Journal of Sound and Vibration 27 (2) (1973) 235-260. 
[30] D. J. Mead, A new method of analyzing wave propagation in periodic structures; applications to periodic timoshenko beams and stiffened plates, Journal of Sound and Vibration 104 (1) (1986) 9-27.

[31] D. J. Mead, The forced vibration of one-dimensional multi-coupled periodic structures: An application to finite element analysis, Journal of Sound and Vibration 319 (1-2) (2009) 282-304.

[32] D. Mead, S. Parthan, Free wave propagation in two-dimensional periodic plates, Journal of Sound and Vibration 64 (3) (1979) 325-348.

[33] D. Rašković, On some characteristics of the frequency equation of torsional vibrations of light shafts with several disks, Publications de l'Institut Mathématique 5 (11) (1953) 155-164.

[34] D. P. Raškovic, On some characteristics of the frequency equations of small vibrations of some particular holonomic conservative systems, The Quarterly Journal of Mechanics and Applied Mathematics 9 (3) (1956) $334-344$.

[35] M. Dublin, H. R. Friedrich, Forced responses of two elastic beams interconnected by spring-damper systems, Journal of the Aeronautical Sciences 23 (9) (1956) 824-829.

[36] J. Seelig, W. Hoppmann, Normal mode vibrations of systems of elastically connected parallel bars, The Journal of the Acoustical Society of America 36 (1) (1964) 93-99.

[37] S. Chonan, Dynamical behaviours of elastically connected double-beam systems subjected to an impulsive load, Bulletin of JSME 19 (132) (1976) 595-603.

[38] T. R. Hamada, H. Nakayama, K. Hayashi, Free and forced vibrations of elastically connected double-beam systems, Bulletin of JSME 26 (221) (1983) 1936-1942.

[39] Z. Oniszczuk, Free transverse vibrations of elastically connected simply supported double-beam complex system, Journal of sound and vibration 232 (2) (2000) 387-403.

[40] Z. Oniszczuk, Forced transverse vibrations of an elastically connected complex simply supported double-beam system, Journal of Sound and Vibration 264 (2) (2003) 273-286.

[41] S. G. Kelly, S. Srinivas, Free vibrations of elastically connected stretched beams, Journal of Sound and Vibration $326(3-5)(2009) 883-893$.

[42] S. G. Kelly, Free and forced vibrations of elastically connected structures, Advances in Acoustics and Vibration 2010.

[43] Q. Mao, Free vibration analysis of elastically connected multiple-beams by using the adomian modified decomposition method, Journal of Sound and Vibration 331 (11) (2012) 2532-2542. 
[44] V. Stojanović, P. Kozić, G. Janevski, Exact closed-form solutions for the natural frequencies and stability of elastically connected multiple beam system using timoshenko and high-order shear deformation theory, Journal of sound and vibration 332 (3) (2013) 563-576.

[45] D. Karličić, T. Murmu, M. Cajić, P. Kozić, S. Adhikari, Dynamics of multiple viscoelastic carbon nanotube based nanocomposites with axial magnetic field, Journal of Applied Physics 115 (23) (2014) 234303.

[46] I. R. Pavlović, D. Karličić, R. Pavlović, G. Janevski, I. Ćirić, Stochastic stability of multi-nanobeam systems, International Journal of Engineering Science 109 (2016) 88-105.

[47] B. Sudret, Meta-models for structural reliability and uncertainty quantification, in: Proceedings of 5th AsianPacific Symposium on Stuctural Reliabilty and its Applications (APSSRA, 2012), Singapore, 2012, pp. 53-76. arXiv:1203.2062.

[48] J. Vanhatalo, J. Riihimaki, J. Hartikainen, P. Jylanki, V. Tolvanen, A. Vehtari, GPstuff : Bayesian Modeling with Gaussian Processes, Journal of Machine Learning Research 14 (2013) 1175-1179.

[49] V. Dubourg, Adaptive surrogate models for reliability analysis and reliability-based design optimization, Ph.D. thesis, PhD thesis, Universite Blaise Pascal, Clermont-Ferrand, France (2011).

[50] S. E. Pryse, A. Kundu, S. Adhikari, Projection methods for stochastic dynamic systems: A frequency domain approach, Computer Methods in Applied Mechanics and Engineering 338 (2018) 412-439.

[51] M. Ainsworth, J. Oden, A posteriori error estimation in finite element analysis, Computer Methods in Applied Mechanics and Engineering 142 (1-2) (1997) 1-88.

[52] D. G. Krige, A Statistical approach to some basic mine valuation problems on the witwatersrand, Journal of the Chemical, Metallurgical and Mining Society of South Africa 52 (6) (1951) 119 - 139.

[53] J. Sacks, W. Welch, T. Mitchell, H. Wynn, Design and analysis of computer experiments, Statistical Science 4 (4) (1989) 409-423.

[54] H. H. Khodaparast, J. E. Mottershead, K. J. Badcock, Interval model updating with irreducible uncertainty using the Kriging predictor, Mechanical Systems and Signal Processing 25 (4) (2011) 1204-1226.

[55] T. Chatterjee, R. Chowdhury, Adaptive Bilevel Approximation Technique for Multiobjective Evolutionary Optimization, Journal of Computing in Civil Engineering (2016) 04016071(1-16)doi:10.1061/(ASCE)CP.19435487.0000643.

[56] T. Chatterjee, R. Chowdhury, P. Ramu, Decoupling Uncertainty Quantification from Robust Design Optimization, Structural and Multidisciplinary Optimization 59 (2019) 1969-1990. 
[57] R. Nayek, S. Chakraborty, S. Narasimhan, A Gaussian process latent force model for joint input-state estimation in linear structural systems, Mechanical Systems and Signal Processing 128 (2019) 497-530.

[58] S. Lophaven, H. Nielson, J. Sondergaard, DACE A MATLAB Kriging Toolbox, Tech. rep., Technical University of Denmark, IMM-TR-2002-12, Technical University of Denmark (2002).

[59] M. Moustapha, J. M. Bourinet, B. Guillaume, B. Sudret, Comparative Study of Kriging and Support Vector Regression for Structural Engineering Applications, Journal of Uncertainty in Engineering Systems, Part A: Civil Engineering 4 (2) (2018) 04018005.

[60] T. Chatterjee, S. Chakraborty, R. Chowdhury, A bi-level approximation tool for the computation of FRFs in stochastic dynamic systems, Mechanical Systems and Signal Processing 70-71 (2016) 484-505.

[61] D. R. Jones, A taxonomy of global optimization methods based on response surfaces, Journal of Global Optimization 21 (4) (2001) 345-383.

[62] T. Chatterjee, R. Chowdhury, h âĂŞ p adaptive model based approximation of moment free sensitivity indices, Computer Methods in Applied Mechanics and Engineering 332 (2018) 572-599.

[63] N. J. Higham, Functions of Matrices: Theory and Computation, SIAM, 2008.

[64] D. J. Higham, N. J. Higham, MATLAB Guide, third edit Edition, SIAM, 2017.

[65] J. Reddy, An introduction to the finite element method, Vol. 1221, McGraw-Hill New York, USA, 2004.

[66] M. McKay, R. J. Beckman, W. J. Conover, A comparison of three methods for selecting values of input variables in the analysis of output from a computer code, Technometrics 21 (2) (1979) 239-245.

[67] A. Gallina, L. Pichler, T. Uhl, Enhanced meta-modelling technique for analysis of mode crossing, mode veering and mode coalescence in structural dynamics, Mechanical Systems and Signal Processing 25 (7) (2011) 22972312.

\section{Appendix 1: System matrices in Example 2}

When the multi-beam-mass system consists of three beams and three attached masses, i.e. $m=3$, the system matrices take the form:

$$
\mathbf{K}=\left[\begin{array}{ccc}
\mathbf{K}_{b} & \mathbf{0} & \mathbf{0} \\
\mathbf{0} & \mathbf{K}_{b} & \mathbf{0} \\
\mathbf{0} & \mathbf{0} & \mathbf{K}_{b}
\end{array}\right]+\left[\begin{array}{ccc}
\mathbf{C}_{e l} & -\mathbf{C}_{e l} & \mathbf{0} \\
-\mathbf{C}_{e l} & 2 \mathbf{C}_{e l} & -\mathbf{C}_{e l} \\
\mathbf{0} & -\mathbf{C}_{e l} & \mathbf{C}_{e l}
\end{array}\right]
$$




$$
\mathbf{M}=\left[\begin{array}{ccc}
\mathbf{M}_{b} & \mathbf{0} & \mathbf{0} \\
\mathbf{0} & \mathbf{M}_{b} & \mathbf{0} \\
\mathbf{0} & \mathbf{0} & \mathbf{M}_{b}
\end{array}\right]+\left[\begin{array}{ccc}
\mathbf{R}_{m} & \mathbf{0} & \mathbf{0} \\
\mathbf{0} & \mathbf{R}_{m} & \mathbf{0} \\
\mathbf{0} & \mathbf{0} & \mathbf{R}_{m}
\end{array}\right]
$$

The sub-matrices $\mathbf{K}_{b}$ and $\mathbf{M}_{b}$ correspond to the global stiffness and mass matrices for a single beam, and the submatrices $\mathbf{C}_{e l}$ and $\mathbf{R}_{m}$ are related to the additional stiffness and mass in the MBS due to the additional masses and coupling stiffnesses. Thus

$$
\mathbf{K}_{b}=\underset{e=1}{n_{e l e}^{\mathcal{A}}} \mathbf{K}^{e}, \quad \mathbf{M}_{b}=\underset{e=1}{n_{\text {ele }}^{\mathcal{A}}} \mathbf{M}^{e}, \quad \mathbf{C}_{e l}=\underset{e=1}{n_{\text {ele }}^{\mathcal{A}}} \mathbf{C}^{e}, \quad \mathbf{R}_{m}=\underset{e=1}{n_{\text {ele }}^{\mathcal{A}}} \mathbf{R}^{e}
$$

where $n_{\text {ele }}$ is number elements per beam in the MBS system, and $\mathbf{K}^{e}, \mathbf{M}^{e}$ and $\mathbf{K}^{e}$ are given by

$$
\begin{gathered}
K_{i j}^{e}=\int_{x_{e}}^{x_{e+1}} E I \phi_{i}^{\prime \prime} \phi_{j}^{\prime \prime} d x, \quad M_{i j}^{e}=\int_{x_{e}}^{x_{e+1}} \rho A \phi_{i} \phi_{j} d x \\
C_{i j}^{e}=\int_{x_{e}}^{x_{e+1}} k \phi_{i} \phi_{j} d x, \quad R_{i j}^{e}=\int_{x_{e}}^{x_{e+1}} \sum_{p=1}^{N} m_{p} \delta\left(x-a_{p}\right) \phi_{i} \phi_{j} d x .
\end{gathered}
$$

